\title{
Tamari Lattices for Parabolic Quotients of the Symmetric Group
}

\author{
Henri Mühle* \\ Institut für Algebra \\ Technische Universität Dresden \\ Dresden, Germany \\ henri.muehle@tu-dresden.de
}

\author{
Nathan Williams ${ }^{\dagger}$ \\ University of Texas at Dallas \\ Dallas, U.S.A. \\ nathan.f.williams@gmail.com
}

Submitted: May 4, 2018; Accepted: Oct 28, 2019; Published: Nov 22, 2019

(C) The authors. Released under the CC BY-ND license (International 4.0).

\begin{abstract}
We generalize the Tamari lattice by extending the notions of 231-avoiding permutations, noncrossing set partitions, and nonnesting set partitions to parabolic quotients of the symmetric group $\mathfrak{S}_{n}$. We show bijectively that these three objects are equinumerous. We show how to extend these constructions to parabolic quotients of any finite Coxeter group. The main ingredient is a certain aligned condition of inversion sets; a concept which can in fact be generalized to any reduced expression of any element in any (not necessarily finite) Coxeter group.
\end{abstract}

Mathematics Subject Classifications: 20F55, 06A07, 52C35

\section{Introduction}

\subsection{Parabolic Tamari Lattices}

The Tamari lattice $\mathcal{T}_{n}$ was introduced by D. Tamari as a partial order encoding the associativity of the Catalan-many binary bracketings of a word of length $n+1$ [31]. The weak order Weak $\left(\mathfrak{S}_{n}\right)$ on the group of permutations $\mathfrak{S}_{n}$ is the oriented Cayley graph of $\mathfrak{S}_{n}$, using the generating set $S$ of adjacent transpositions. Rephrasing slightly, A. Björner and M. Wachs realized $\mathcal{T}_{n}$ as a sublattice of Weak $\left(\mathfrak{S}_{n}\right)$ by considering the subset $\mathfrak{S}_{n}(231) \subseteq \mathfrak{S}_{n}$ of 231-avoiding permutations, whose inversions sets they characterize as "compressed" [7, Section 9]. N. Reading extended this result by noting that $\mathcal{T}_{n}$ was a lattice quotient of $\operatorname{Weak}\left(\mathfrak{S}_{n}\right)[23]$.

${ }^{*} \mathrm{HM}$ was partially supported by a Public Grant overseen by the French National Research Agency (ANR) as part of the "Investissements d'Avenir" Program (Reference: ANR-10-LABX-0098), and by Digiteo project PAAGT (Nr. 2015-3161D).

${ }^{\dagger}$ NW was partially supported by a Simons Foundation Collaboration Grant. 
We generalize the Tamari lattice from the symmetric group to its parabolic quotients. Any $J \subseteq S$ defines the parabolic quotient $\mathfrak{S}_{n}^{J}$, consisting of those permutations $w \in \mathfrak{S}_{n}$ with descents only at positions not in $J$ - that is, if $w(i)>w(i+1)$, then $s_{i} \notin J$. Parabolic quotients have a weak order $\operatorname{Weak}\left(\mathfrak{S}_{n}^{J}\right)$ inherited from Weak $\left(\mathfrak{S}_{n}\right)$. We specify a subset $\mathfrak{S}_{n}^{J}(231) \subseteq \mathfrak{S}_{n}^{J}$ by introducing a generalized notion of 231-avoidance, dependent on $J$, which can again be seen as a "compressed" condition on inversion sets.

Theorem 1. Let $n>0$. For $J \subseteq S$, the restriction $\operatorname{Weak}\left(\mathfrak{S}_{n}^{J}\right)$ to $\mathfrak{S}_{n}^{J}(231)$ is a lattice, which we denote $\mathcal{T}_{n}^{J}$. Although $\mathcal{T}_{n}^{J}$ is not generally a sublattice of Weak $\left(\mathfrak{S}_{n}^{J}\right)$, it is a lattice quotient of $\operatorname{Weak}\left(\mathfrak{S}_{n}^{J}\right)$.

When $J=\emptyset$, we recover the Tamari lattice on 231-avoiding permutations, and we therefore refer to $\mathcal{T}_{n}^{J}$ as the parabolic Tamari lattice. ${ }^{1}$

\subsection{Parabolic Catalan Objects}

In recent years, many combinatorial families enumerated by the Catalan numbers have been linked to the symmetric group. Two prototypical examples for this phenomenon are the noncrossing set partitions - which are the elements in an interval in the absolute order for $\mathfrak{S}_{n}$ - and the nonnesting set partitions - which are order ideals in the root poset of $\mathfrak{S}_{n}$. We propose new generalizations of noncrossing and nonnesting partitions to $\mathfrak{S}_{n}^{J}$, and denote the resulting sets by $\mathrm{NC}_{n}^{J}$ and $\mathrm{NN}_{n}^{J}$, respectively. For $J=\emptyset$, we recover the classical noncrossing and nonnesting set partitions, respectively.

The well-known property that the 231-avoiding permutations, noncrossing and nonnesting partitions are equinumerous, survives our generalization to parabolic quotients.

Theorem 2. For $n>0$ and $J \subseteq S$, we have bijections

$$
\mathfrak{S}_{n}^{J}(231) \simeq \mathrm{NC}_{n}^{J} \simeq \mathrm{NN}_{n}^{J}
$$

Although we no longer have a nice closed formula in general, the parabolic nonnesting partitions enable us to write down a determinantal formula for the parabolic Catalan numbers, see the end of Section 5.

\subsection{Generalizations to Finite Coxeter Groups}

A. Björner and M. Wachs' observation that the Tamari lattice arises as a sublattice of the weak order on $\mathfrak{S}_{n}$ was the precursor to N. Reading's definition of the Cambrian lattices. Fixing a Coxeter element $c$, such a lattice may be described as the restriction of the weak order of a finite Coxeter group to certain c-aligned elements, which - as with 231-avoiding permutations - are characterized by their inversion sets [23]. The Cambrian lattices thus naturally generalize the Tamari lattice to any finite Coxeter group and any Coxeter element.

\footnotetext{
${ }^{1}$ This nomenclature is slightly ambiguous, since it could refer to either parabolic quotients or parabolic subgroups - but the Tamari lattice of a parabolic subgroup is a direct product of Tamari lattices, and so deserves no special name.
} 
N. Reading's aligned elements of a Coxeter group $W$ have a surprisingly different characterization using reduced words, and in the guise of sortable elements they provide a bridge between two famous families of objects attached to a finite Coxeter group: the $W$ noncrossing partitions and the $W$-clusters [24]. Remarkably, these objects are uniformly enumerated by a simple product formula depending on the degrees of $W$ [28, Remark 2].

In the second part of this article, we define analogues of the sets $\mathfrak{S}_{n}^{J}(231), \mathrm{NC}_{n}^{J}$ and $\mathrm{NN}_{n}^{J}$ for all finite Coxeter groups, and we study in which cases we retain the property that these sets are equinumerous. We present computational evidence that this is the case for groups of "coincidental" type $A_{n}, B_{n}, H_{3}$, and $I_{2}(m)$ (these are exactly those types for which every wall of the Coxeter complex is again a Coxeter complex).

\subsection{Further Generalizations}

The key idea in the definition of parabolic alignment is a certain forcing of inversions with respect to the root order of a particular reduced expression for the longest element in the parabolic quotient. In the last part of this article we generalize this idea to any reduced expression of any element of any (not necessarily finite) Coxeter group. This generalization comes at the price of losing the lattice property.

\subsection{Outline of the Paper}

This article is structured as follows. We recall the basic notions for the symmetric group and its parabolic quotients in Section 2. In Section 3 we define (J,231)-avoiding permutations and characterize them in terms of their inversion sets. We prove Theorem 1 in Section 3.4. Sections 4 and 5 introduce noncrossing partitions and nonnesting partitions for parabolic quotients of the symmetric group, and culminate in the proof of Theorem 2 . Section 6 is concerned with the generalization of 231-avoiding permutations, noncrossing and nonnesting partitions to parabolic quotients of arbitrary finite Coxeter groups. We also propose a definition of a parabolic Coxeter-Catalan number for the coincidental types at the end of Section 6.6. In Section 6.7 we generalize the definition of alignment to any reduced expression of any element in any (not-necessarily finite) Coxeter group.

\section{The Symmetric Group}

In this section, we recall the definitions of weak order, 231-avoiding permutations, and parabolic quotients of the symmetric group.

\subsection{Weak Order}

The symmetric group $\mathfrak{S}_{n}$ is the group of permutations of $[n]:=\{1,2, \ldots, n\}$. Let $S:=$ $\left\{s_{1}, s_{2}, \ldots, s_{n-1}\right\}$ denote the set of adjacent transpositions of $\mathfrak{S}_{n}$, i.e. $s_{i}:=(i, i+1)$ for $i \in[n-1]$. It is well known that $\mathfrak{S}_{n}$ is isomorphic to the Coxeter group $A_{n-1}$, and so admits a presentation of the form

$$
\left.\mathfrak{S}_{n}=\langle S| s_{i}^{2}=\left(s_{i} s_{j}\right)^{2}=\left(s_{i} s_{i+1}\right)^{3}=e, \text { for }|i-j|>1\right\rangle
$$


where $e$ denotes the identity permutation. We may specify a permutation $w \in \mathfrak{S}_{n}$ using one-line notation: $w=w_{1} w_{2} \ldots w_{n}$, where $w_{i}=w(i)$ for $i \in[n]$. Its inversion set is defined by

$$
\operatorname{Inv}(w):=\left\{(i, j) \mid 1 \leqslant i<j \leqslant n \text { and } w_{i}>w_{j}\right\} .
$$

The (left) weak order is the partial order on $\mathfrak{S}_{n}$ defined by $u \leqslant_{S} v$ if and only if $\operatorname{Inv}(u) \subseteq \operatorname{Inv}(v)$; and we denote by $\operatorname{Weak}\left(\mathfrak{S}_{n}\right)$ the partially ordered set $\left(\mathfrak{S}_{n}, \leqslant_{S}\right)$. The cover relations of $\operatorname{Weak}\left(\mathfrak{S}_{n}\right)$ are relations $u \leqslant_{S} v$ such that $\operatorname{Inv}(v) \backslash \operatorname{Inv}(u)=\{(i, j)\}$ with $v_{i}=v_{j}+1$. We usually write $u \lessdot_{S} v$ in such a case. The poset Weak $\left(\mathfrak{S}_{n}\right)$ is a lattice [5, Theorem 3.2.1], so that any two elements have a greatest lower bound and a least upper bound. In particular, there is a unique maximal element $w_{\circ}$ in Weak $\left(\mathfrak{S}_{n}\right)$ whose one-line notation is $w_{\circ}=n(n-1) \ldots 1$. We refer the reader to [5, Section 3] for more background on the weak order, in the broader context of Coxeter groups.

A permutation $w \in \mathfrak{S}_{n}$ is 231-avoiding if there exists no triple $i<j<k$ such that $w_{k}<w_{i}<w_{j}$. Let $\mathfrak{S}_{n}(231)$ denote the set of 231-avoiding permutations of $\mathfrak{S}_{n}$. Lemma 9.8 in [7] implies that the 231-avoiding permutations can be characterized by their inversion sets. More precisely, $w \in \mathfrak{S}_{n}$ is 231 -avoiding if and only if its inversion set is compressed, i.e. if $i<j<k$ and $(i, k) \in \operatorname{Inv}(w)$, then $(i, j) \in \operatorname{Inv}(w)$.

Remark 3. Let $w \in \mathfrak{S}_{n}$ and choose $i<j<k$. It is immediate to verify that whenever $(i, k) \in \operatorname{Inv}(w)$, then we also have $(i, j) \in \operatorname{Inv}(w)$ or $(j, k) \in \operatorname{Inv}(w)$ (or both). We may interpret the property that $\operatorname{Inv}(w)$ is compressed as stating that $\operatorname{Inv}(w)$ is aligned with respect to the lexicographic order on all transpositions. This perspective foreshadows N. Reading's definition of aligned elements in a Coxeter group [24, Section 4]. We generalize this notion in Definitions 33 and 43.

The next result identifies the Tamari lattice $\mathcal{T}_{n}$ as the subposet of the weak order on $\mathfrak{S}_{n}$ induced by the 231 -avoiding permutations. The reader may take this as the definition of $\mathcal{T}_{n}$.

Theorem 4 ([7, Theorem 9.6(ii)]). For $n>0$ the poset Weak $\left(\mathfrak{S}_{n}(231)\right)$ is isomorphic to the Tamari lattice $\mathcal{T}_{n}$.

\subsection{Parabolic Quotients}

Any subset $J \subseteq S$ naturally generates a subgroup of $\mathfrak{S}_{n}$ isomorphic to a direct product of symmetric groups of smaller rank. We call such a subgroup parabolic, and we denote it by $\left(\mathfrak{S}_{n}\right)_{J}$. We define the parabolic quotient of $\mathfrak{S}_{n}$ with respect to $J$ by

$$
\mathfrak{S}_{n}^{J}:=\left\{w \in \mathfrak{S}_{n} \mid w<_{S} w s \text { for all } s \in J\right\} .
$$

The set $\mathfrak{S}_{n}^{J}$ thus consists of the minimal length representatives of the right cosets of the corresponding parabolic subgroup. By [5, Proposition 2.4.4], any permutation $w \in \mathfrak{S}_{n}$ can be uniquely written as $w=w^{J} \cdot w_{J}$, for some $w^{J} \in \mathfrak{S}_{n}^{J}$ and $w_{J} \in\left(\mathfrak{S}_{n}\right)_{J}$. In particular, the maximal element $w_{\circ} \in \mathfrak{S}_{n}$ is written as $w_{\circ}=w_{\circ}^{J} \cdot\left(w_{\circ}\right)_{J}$, where $w_{\circ}^{J}$ is the longest element of $\mathfrak{S}_{n}^{J}$ and $\left(w_{\circ}\right)_{J}$ is the longest element of $\left(\mathfrak{S}_{n}\right)_{J}$. 
Since any element of $\mathfrak{S}_{n}^{J}$ is itself a permutation, we may consider $\operatorname{Weak}\left(\mathfrak{S}_{n}^{J}\right)$ - the restriction of the weak order on $\mathfrak{S}_{n}$ to the parabolic quotient. It follows from [6, Theorem 4.1] that this poset is isomorphic to the weak order interval $\left[e, w_{\circ}^{J}\right]$, and is therefore a lattice.

\section{Tamari Lattices for Parabolic Quotients of $\mathfrak{S}_{n}$}

In this section, we define the set $\mathfrak{S}_{n}^{J}(231)$ of 231 -avoiding permutations of the parabolic quotient $\mathfrak{S}_{n}^{J}$. We characterize the inversion sets of the permutations in $\mathfrak{S}_{n}^{J}(231)$ and prove that $\operatorname{Weak}\left(\mathfrak{S}_{n}^{J}(231)\right)$ is a lattice.

\subsection{1-Avoidance}

Let $J:=S \backslash\left\{s_{j_{1}}, s_{j_{2}}, \ldots, s_{j_{r}}\right\}$, and let $\mathrm{B}(J)$ be the set partition of $[n]$ given by the parts

$$
\left\{\left\{1, \ldots, j_{1}\right\},\left\{j_{1}+1, \ldots, j_{2}\right\}, \ldots,\left\{j_{r-1}+1, \ldots, j_{r}\right\},\left\{j_{r}+1, \ldots, n\right\}\right\} .
$$

We call the parts of $\mathrm{B}(J)$ the J-regions. We indicate the parts of $\mathrm{B}(J)$ occuring in the one-line notation of a permutation $w \in \mathfrak{S}_{n}^{J}$ by vertical bars.

Lemma 5. If $w \in \mathfrak{S}_{n}^{J}$, then the one-line notation of $w$ has the form

$$
w=w_{1}<\cdots<w_{j_{1}}\left|w_{j_{1}+1}<\cdots<w_{j_{2}}\right| \cdots \mid w_{j_{r}+1}<\cdots<w_{n} .
$$

Proof. If this were not the case, then there would be some index $i \in[n]$ such that $w_{i}>$ $w_{i+1}$, and $j_{l}+1<i<j_{l+1}-1$ for some $l \in\{0,1, \ldots, r\}$, where $j_{0}=1$ and $j_{r+1}=n$. It follows that $(i, i+1) \in \operatorname{Inv}(w)$ and $(i, i+1) \notin \operatorname{Inv}\left(w s_{i}\right)$. By definition, it follows that $w s_{i}<_{S} w$, which contradicts the assumption that $w \in \mathfrak{S}_{n}^{J}$, since $s_{i} \in J$.

Definition 6. A permutation $w \in \mathfrak{S}_{n}^{J}$ contains a $(J, 231)$-pattern if there exist three indices $i<j<k$, all of which lie in different $J$-regions, such that $w_{k}<w_{i}<w_{j}$ and $w_{i}=w_{k}+1$. We say that $w$ is $(J, 231)$-avoiding if it does not contain a $(J, 231)$-pattern; and we denote the set of all $(J, 231)$-avoiding permutations of $\mathfrak{S}_{n}^{J}$ by $\mathfrak{S}_{n}^{J}(231)$.

Example 7. The left image in Figure 1 shows $\operatorname{Weak}\left(\mathfrak{S}_{4}^{\left\{s_{2}\right\}}\right)$, where the $\left(\left\{s_{2}\right\}, 231\right)$ avoiding permutations have been shaded in gray. Notice that the longest permutation $4|23| 1$ is not 231-avoiding, since it contains the subsequence 231. However, since the 2 and the 3 lie in the same $\left\{s_{2}\right\}$-region, this sequence does not form an $\left(\left\{s_{2}\right\}, 231\right)$-pattern.

The following Theorem 8 shows that Definition 6 is a generalization of 231-avoiding permutations by showing that $\mathfrak{S}_{n}^{\emptyset}(231)=\mathfrak{S}_{n}(231)$.

Proposition 8. If $w \in \mathfrak{S}_{n}$ has a 231-pattern, then there exist indices $i<j<k$ such that $w_{k}<w_{i}<w_{j}$ and $w_{i}=w_{k}+1$. Consequently, $\mathfrak{S}_{n}^{\emptyset}(231)=\mathfrak{S}_{n}(231)$. 


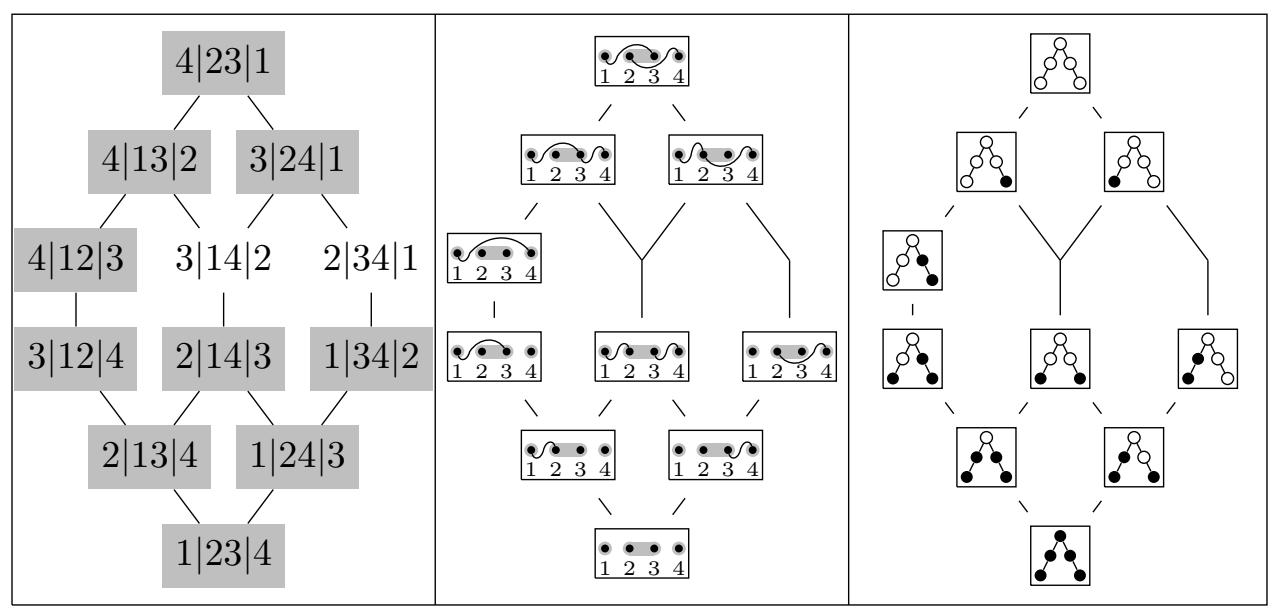

Figure 1: The parabolic Tamari lattice $\mathcal{T}_{4}^{\left\{s_{2}\right\}}$. The poset on the left has every permutation of the parabolic quotient $\mathfrak{S}_{4}^{\left\{s_{2}\right\}}$ with the $\left(\left\{s_{2}\right\}, 231\right)$-avoiding elements marked in gray; the posets in the middle and on the right are labeled by the $\left\{s_{2}\right\}$-noncrossing partitions and the $\left\{s_{2}\right\}$-nonnesting partitions of a four-element set, respectively.

Proof. Let $i<j<k$ be indices such that $w_{k}<w_{i}<w_{j}$, and choose them in such a way that $w_{i}-w_{k}$ is minimal. We claim that in this case $w_{i}=w_{k}+1$. Assume the opposite. Then there is some $d \in[n]$ with $w_{k}<w_{d}<w_{i}$. If $d<j$, then $(d, j, k)$ forms a 231-pattern in $w$. But $w_{d}-w_{k}<w_{i}-w_{k}$, which contradicts the choice of $(i, j, k)$. If $d>j$, then $(i, j, d)$ forms a 231-pattern in $w$. But $w_{i}-w_{d}<w_{i}-w_{k}$, which again contradicts the choice of $(i, j, k)$.

Remark 9. After an extended abstract of this paper appeared in [19], R. Proctor and M. Willis gave a different definition of parabolic pattern avoidance [22]. More precisely, if $R=\left\{j_{1}, j_{2}, \ldots, j_{r}\right\}$, then they say that $w \in \mathfrak{S}_{n}^{J}$ is $R$-312-containing if there exists $h \in[r-1]$ and indices $a, b, c \in[n]$ with $a \leqslant j_{h}<b \leqslant j_{h+1}<c$ such that $w_{b}<$ $w_{c}<w_{a}$. Any element of $\mathfrak{S}_{n}^{J}$ that is not $R$-312-containing is $R$-312-avoiding. They suggested the term "parabolic Catalan number" for the cardinality of the set of all $R$ 312-avoiding permutations, and exhibit several combinatorial objects associated with $\mathfrak{S}_{n}^{J}$ that are enumerated by these numbers [20-22].

It is straightforward to define $R$-231-avoiding permutations in the sense of $\mathrm{R}$. Proctor and M. Willis, but this definition is more restrictive than our Definition 6. For example, the permutation $3|24| 1 \in \mathfrak{S}_{4}^{\left\{s_{2}\right\}}$ is $\{1,3\}$-231-containing, since the ' 3 ', the ' 4 ', and the ' 1 ' form a parabolic 231-pattern. However, the ' 3 ' and the ' 1 ' do not form a descent, and this permutation turns out to be $\left(\left\{s_{2}\right\}, 231\right)$-avoiding. 


\subsection{Compressed Inversion Sets}

We now generalize A. Björner and M. Wachs' definition of compressed inversion sets to parabolic quotients. Define the descent set of $w$ by

$$
\operatorname{Des}(w):=\left\{(i, j) \in \operatorname{Inv}(w) \mid w_{i}=w_{j}+1\right\} .
$$

Definition 10. An inversion $\operatorname{set} \operatorname{Inv}(w)$ for a permutation $w \in \mathfrak{S}_{n}^{J}$ is $J$-compressed if whenever there are three indices $i<j<k$, each in different $J$-regions and such that $(i, k) \in \operatorname{Des}(w)$, it follows that $(i, j) \in \operatorname{Inv}(w)$.

Lemma 11. A permutation $w \in \mathfrak{S}_{n}^{J}$ is (J,231)-avoiding if and only if $\operatorname{Inv}(w)$ is $J$ compressed.

Proof. Suppose first that $w \in \mathfrak{S}_{n}^{J}$ is not $(J, 231)$-avoiding. By definition there exist indices $i<j<k$ each in different $J$-regions such that $w_{k}<w_{i}<w_{j}$ as well as $w_{i}=w_{k}+1$. This means that $(i, k) \in \operatorname{Des}(w)$ but $(i, j) \notin \operatorname{Inv}(w)$, which implies that $\operatorname{Inv}(w)$ is not $J$-compressed.

On the other hand, suppose that $w$ is $(J, 231)$-avoiding, and choose three indices $i<j<k$, each in different $J$-regions and such that $(i, k) \in \operatorname{Des}(w)$. Since $w$ does not contain a $(J, 231)$-pattern we must have $w_{j}<w_{i}$, which implies $(i, j) \in \operatorname{Inv}(w)$. Hence $\operatorname{Inv}(w)$ is $J$-compressed.

\subsection{Tamari Lattices for Parabolic Quotients}

Write Weak $\left(\mathfrak{S}_{n}^{J}(231)\right)$ for the restriction of the weak order on the parabolic quotient $\mathfrak{S}_{n}^{J}$ to the $(J, 231)$-avoiding permutations. We now prove the first part of Theorem 1 - that Weak $\left(\mathfrak{S}_{n}^{J}(231)\right)$ is a lattice. The proof follows from the next lemma, which is modeled after [23, Lemma 5.6] for the case $J=\emptyset$.

Lemma 12. For every $w \in \mathfrak{S}_{n}^{J}$, there is a unique $w^{\prime} \in \mathfrak{S}_{n}^{J}(231)$ such that $\operatorname{Inv}\left(w^{\prime}\right)$ is the maximal set under containment among all $J$-compressed inversion sets $\operatorname{Inv}(u) \subseteq \operatorname{Inv}(w)$.

Proof. We proceed by induction on the cardinality of $\operatorname{Inv}(w)$. If $\operatorname{Inv}(w)=\emptyset$, then $\operatorname{Inv}(w)$ is $J$-compressed and the claim holds trivially. Suppose that $|\operatorname{Inv}(w)|=r$, and that the claim is true for all $x \in \mathfrak{S}_{n}^{J}$ with $|\operatorname{Inv}(x)|<r$.

If $\operatorname{Inv}(w)$ is already $J$-compressed, then we set $w^{\prime}=w$ and we are done. Otherwise, Lemma 11 implies that $w$ contains an instance of a $(J, 231)$-pattern, which means that there are indices $i<j<k$ that all lie in different $J$-regions such that $w_{k}<w_{i}<w_{j}$ and $w_{i}=w_{k}+1$. Consider the lower cover $u$ of $w$ in which $w_{i}$ and $w_{k}$ are exchanged. In particular, we have $\operatorname{Inv}(w)=\operatorname{Inv}(u) \cup\{(i, k)\}$. By the induction hypothesis, there exists some $u^{\prime} \in \mathfrak{S}_{n}^{J}$ such that $\operatorname{Inv}\left(u^{\prime}\right)$ is the unique maximal $J$-compressed inversion set that is contained in $\operatorname{Inv}(u)$. We claim that $w^{\prime}=u^{\prime}$.

In order to prove this claim, we choose some element $v \in \mathfrak{S}_{n}^{J}$ such that $\operatorname{Inv}(v)$ is $J$-compressed and $\operatorname{Inv}(v) \subseteq \operatorname{Inv}(w)$. By construction, we have $(i, j) \notin \operatorname{Inv}(w)$, and hence $(i, j) \notin \operatorname{Inv}(v)$. Since $\operatorname{Inv}(v)$ is $J$-compressed it follows by definition that $v_{i} \neq v_{k}+1$. 
We want to show that $\operatorname{Inv}(v) \subseteq \operatorname{Inv}(u)$, which amounts to showing that $(i, k) \notin \operatorname{Inv}(v)$ because $\operatorname{Inv}(w) \backslash \operatorname{Inv}(u)=\{(i, k)\}$.

We assume the opposite, and in view of the argument above it follows that $v_{i}>v_{k}+1$. Let $d$ be the index such that $v_{d}=v_{k}+1$, and let $e$ be the index such that $v_{i}=v_{e}+1$. Since $w_{i}=w_{k}+1$, we observe the following:

$$
\begin{array}{lll}
\text { either } & w_{d}<w_{k} \quad \text { or } \quad w_{i}<w_{d}, \quad \text { and } \\
\text { either } \quad w_{e}<w_{k} & \text { or } \quad w_{i}<w_{e} .
\end{array}
$$

We have the following relations:

$$
v_{j}>v_{i}>v_{e} \geqslant v_{d}>v_{k} .
$$

(If $v_{j}<v_{i}$, then $(i, j) \in \operatorname{Inv}(v) \subseteq \operatorname{Inv}(w)$, which is a contradiction.) We now distinguish five cases.

(i) Let $d<i<k$. Then $(d, k) \in \operatorname{Des}(v) \subseteq \operatorname{Inv}(w)$. It follows that $w_{d}>w_{k}$, and (D) implies $w_{d}>w_{i}$. Lemma 5 implies that $d$ and $i$ lie in different $J$-regions. Since $\operatorname{Inv}(v)$ is $J$-compressed, we conclude $(d, i) \in \operatorname{Inv}(v)$. Hence $v_{i}<v_{d}=v_{k}+1<v_{i}$, which is a contradiction.

(ii) Let $i<d<k$. Then $(i, d),(d, k) \in \operatorname{Inv}(v) \subseteq \operatorname{Inv}(w)$. It follows that $w_{i}>w_{d}>w_{k}$, which contradicts $(i, k) \in \operatorname{Des}(w)$.

(iii) Let $i<e<k$. Then $(i, e),(e, k) \in \operatorname{Inv}(v) \subseteq \operatorname{Inv}(w)$. It follows that $w_{i}>w_{e}>w_{k}$, which contradicts $(i, k) \in \operatorname{Des}(w)$.

(iv) Let $i<k<e$. Then $(i, e) \in \operatorname{Des}(v) \subseteq \operatorname{Inv}(w)$. It follows that $w_{i}>w_{e}$, and (E) implies $w_{e}<w_{k}$. Lemma 5 implies that $k$ and $e$ lie in different $J$-regions. Since $\operatorname{Inv}(v)$ is $J$-compressed, we conclude $(k, e) \in \operatorname{Inv}(v)$. Hence $v_{i}=v_{e}+1<v_{k}+1<v_{i}$, which is a contradiction.

(v) Let $e<i<k<d$, which in particular implies that $(e, d) \in \operatorname{Inv}(v) \subseteq \operatorname{Inv}(w)$. Moreover, $(e, k),(i, d) \in \operatorname{Des}(v) \subseteq \operatorname{Inv}(w)$. It follows that $w_{i}>w_{d}$ as well as $w_{e}>w_{k}$. Now (D) and (E) imply $w_{d}<w_{k}$ and $w_{e}>w_{i}$, respectively. Lemma 5 implies that $e, i, k$ and $d$ all lie in different $J$-regions.

Let $e^{\prime}$ be the smallest element in the $J$-region of $e$ such that $v_{e^{\prime}}>v_{d}$, and let $d^{\prime}$ be the largest element in the $J$-region of $d$ such that $v_{d^{\prime}}<v_{e^{\prime}}$. We record that $e^{\prime} \leqslant e<$ $i<j<k<d \leqslant d^{\prime}$, and we proceed by induction on $v_{e^{\prime}}-v_{d^{\prime}}$. If $v_{e^{\prime}}=v_{d^{\prime}}+1$, then $\left(e^{\prime}, i\right),\left(e^{\prime}, j\right) \in \operatorname{Inv}(v)$, since $\operatorname{Inv}(v)$ is $J$-compressed. Lemma 5 implies that $v_{e} \geqslant v_{e^{\prime}}>$ $v_{j}>v_{i}=v_{e}+1$, which is a contradiction. If $v_{e^{\prime}}>v_{d^{\prime}}+1$, then there must be some index $f$ with $v_{e^{\prime}}>v_{f}>v_{d^{\prime}}+1$. By construction we have $v_{i}=v_{e}+1>v_{e} \geqslant v_{e^{\prime}}>v_{f}$.

If $f<i$ and they do not lie in the same $J$-region, then we can consider the triple $\left(f, i, d^{\prime}\right)$, and obtain a contradiction by induction, since $v_{f}-v_{d^{\prime}}<v_{e^{\prime}}-v_{d^{\prime}}$. If $f>i$ and they do not lie in the same $J$-region, then we can consider the triple $\left(e^{\prime}, i, f\right)$, and obtain a contradiction by induction, since $v_{e^{\prime}}-v_{f}<v_{e^{\prime}}-v_{d^{\prime}}$. If $f$ and $i$ lie in the same $J$-region, then we have $f<i$. We can consider the triple $\left(f, j, d^{\prime}\right)$, and obtain a contradiction by induction, since $v_{f}-v_{d^{\prime}}<v_{e^{\prime}}-v_{d^{\prime}}$.

We have thus shown that $(i, k) \notin \operatorname{Inv}(v)$, which implies $\operatorname{Inv}(v) \subseteq \operatorname{Inv}(u)$. By the induction assumption it follows that $\operatorname{Inv}(v) \subseteq \operatorname{Inv}\left(u^{\prime}\right)$, which proves $w^{\prime}=u^{\prime}$. 
Using Lemma 11, we may reformulate Lemma 12: for every $w \in \mathfrak{S}_{n}^{J}$, there exists a unique maximal $(J, 231)$-avoiding permutation $w^{\prime}$ with $w^{\prime} \leqslant_{S} w$. The following definition gives us notation to refer to $w^{\prime}$.

Definition 13. We define the projection

$$
\Pi_{\downarrow}^{J}: \mathfrak{S}_{n}^{J} \rightarrow \mathfrak{S}_{n}^{J}(231), \quad w \mapsto w^{\prime}
$$

where $w^{\prime}$ is the unique maximal $(J, 231)$-avoiding permutation below $w$.

Proposition 14. The poset $\operatorname{Weak}\left(\mathfrak{S}_{n}^{J}(231)\right)$ is a lattice.

Proof. Let $w_{1}, w_{2} \in \mathfrak{S}_{n}^{J}(231)$. Lemma 12 implies that there exists a unique maximal element $u^{\prime} \in \mathfrak{S}_{n}^{J}(231)$ with $u^{\prime} \leqslant_{S} w_{1}, w_{2}$, which necessarily must be the meet of $w_{1}$ and $w_{2}$ in $\operatorname{Weak}\left(\mathfrak{S}_{n}^{J}(231)\right)$. Since $\operatorname{Inv}\left(w_{\circ}^{J}\right)$ contains all possible inversions, we have $\Pi_{\downarrow}^{J}\left(w_{\circ}^{J}\right)=w_{\circ}^{J}$. We have thus established that Weak $\left(\mathfrak{S}_{n}^{J}(231)\right)$ is a finite meet-semilattice with greatest element $w_{\circ}^{J}$. It is a classical lattice-theoretic result (see for instance [12, Exercise 1.27]) that this suffices to show that Weak $\left(\mathfrak{S}_{n}^{J}(231)\right)$ is a lattice.

Theorem 8 implies that the set $\mathfrak{S}_{n}^{\emptyset}(231)$ coincides with the set of all classical 231avoiding permutations of $\mathfrak{S}_{n}$, and Theorem 4 states that Weak $\left(\mathfrak{S}_{n}^{\emptyset}(231)\right)$ is isomorphic to the Tamari lattice $\mathcal{T}_{n}$. In view of Proposition 14, we denote the poset Weak $\left(\mathfrak{S}_{n}^{J}(231)\right)$ by $\mathcal{T}_{n}^{J}$, and call it the parabolic Tamari lattice.

Remark 15. Consider the parabolic subgroup $\left(\mathfrak{S}_{n}\right)_{J}$, and let $\left(w_{\circ}\right)_{J}$ denote the longest permutation in this subgroup. The poset of all 231-avoiding permutations in the interval $\left[e,\left(w_{\circ}\right)_{J}\right]$ is just an interval in the Tamari lattice $\mathcal{T}_{n}$.

If we consider instead parabolic quotients, then even though the elements in $\mathfrak{S}_{n}^{J}$ form the interval $\left[e, w_{\circ}^{J}\right]$, the lattice $\mathcal{T}_{n}^{J}$ is not an interval in $\mathcal{T}_{n}$. For example, $\mathcal{T}_{4}^{\left\{s_{2}\right\}}$ is depicted in Figure 1. Observe that the maximal element $w_{\circ}^{\left\{s_{2}\right\}}=4|23| 1$ is not 231-avoiding.

Remark 16. The lattice $\mathcal{T}_{n}^{J}$ is not generally a sublattice of $\left[e, w_{\circ}^{J}\right]$. Consider again the case when $n=4$ and $J=\left\{s_{2}\right\}$. Then the meet of $w_{1}=4|13| 2$ and $w_{2}=3|24| 1$ in weak order is $3|14| 2$, while their meet in $\mathcal{T}_{4}^{\left\{s_{2}\right\}}$ is $2|14| 3$.

In certain special cases - for example, when $J=\emptyset$ or for certain $J=S \backslash\{s\}$-we do obtain sublattices.

\subsection{Parabolic Tamari Lattices are Lattice Quotients}

In this section, we prove that $\mathcal{T}_{n}^{J}$ is a lattice quotient of Weak $\left(\mathfrak{S}_{n}^{J}\right)$, completing the proof of Theorem 1. Recall for instance from [23, Section 3] that an equivalence relation $\Theta$ on a lattice is a lattice congruence if and only if all equivalence classes are intervals, and the projections that map an element to the least or greatest element in its equivalence class, respectively, are both order-preserving.

Using the map $\Pi_{\downarrow}^{J}$ from Definition 13 , we define a binary relation $\Theta$ on $\mathfrak{S}_{n}^{J}$ by

$$
\left(w, w^{\prime}\right) \in \Theta \quad \text { if and only if } \Pi_{\downarrow}^{J}(w)=\Pi_{\downarrow}^{J}\left(w^{\prime}\right) .
$$


It is immediate that $\Theta$ is an equivalence relation and $\Pi_{\downarrow}^{J}$ maps $w \in \mathfrak{S}_{n}^{J}$ to the least element in its equivalence class.

Lemma 17. The fibers of $\Pi_{\downarrow}^{J}$ are order-convex, i.e. if $u \leqslant_{S} x \leqslant_{S} v$ and $\Pi_{\downarrow}^{J}(u)=\Pi_{\downarrow}^{J}(v)$, then $\Pi_{\downarrow}^{J}(u)=\Pi_{\downarrow}^{J}(x)$.

Proof. Let $u^{\prime}=\Pi_{\downarrow}^{J}(u)=\Pi_{\downarrow}^{J}(v)$ and $x^{\prime}=\Pi_{\downarrow}^{J}(x)$. Since $x^{\prime} \leqslant_{S} x \leqslant_{S} v$, Lemma 12 implies $x^{\prime} \leqslant_{S} u^{\prime}$. Moreover, since $u^{\prime} \leqslant_{S} u \leqslant_{S} x$, Lemma 12 implies $u^{\prime} \leqslant_{S} x^{\prime}$.

We claim that every equivalence class of $\Theta$ has a greatest element. In view of Lemma 17 this would imply that the equivalence classes of $\Theta$ are intervals in Weak $\left(\mathfrak{S}_{n}^{J}\right)$. In order to describe these greatest elements, we say that a permutation $w \in \mathfrak{S}_{n}^{J}$ has a (J,132)-pattern if there are indices $i<j<k$ each in different $J$-regions such that $w_{i}<w_{k}<w_{j}$ and $w_{k}=w_{i}+1$. We say $w \in \mathfrak{S}_{n}^{J}$ is $(J, 132)$-avoiding if it does not have a $(J, 132)$-pattern. The proof of the following result is almost identical to the proof of Lemma 12.

Lemma 18. For any $w \in \mathfrak{S}_{n}^{J}$, there is a unique minimal (J,132)-avoiding permutation $w^{\prime}$ with $w \leqslant \leqslant_{S} w^{\prime}$.

We therefore obtain a map $\Pi_{\uparrow}^{J}: \mathfrak{S}_{n} \rightarrow \mathfrak{S}_{n}^{J}(132)$ that maps $w$ to the unique minimal (J,132)-avoiding permutation $w^{\prime}$ above $w$.

Lemma 19. The maps $\Pi_{\downarrow}^{J}$ and $\Pi_{\uparrow}^{J}$ are order-preserving.

Proof. We only prove this property for $\Pi_{\downarrow}^{J}$, since the result for $\Pi_{\uparrow}^{J}$ follows analogously.

Let $u, v \in \mathfrak{S}_{n}^{J}$ with $u \leqslant_{S} v$. Lemma 12 states that $\Pi_{\downarrow}^{J}(u) \leqslant_{S} u \leqslant_{S} v$. Since $\Pi_{\downarrow}^{J}(v)$ is maximal among all elements below $v$ with $J$-compressed inversion sets, it follows that $\Pi_{\downarrow}^{J}(u) \leqslant_{S} \Pi_{\downarrow}^{J}(v)$.

Lemma 20. Let $u, v \in \mathfrak{S}_{n}^{J}$ with $u \lessdot_{S} v$. The following are equivalent.

(i) There are indices $i<j<k$, each in different $J$-regions, such that $v_{k}<v_{i}<v_{j}$, $v_{i}=v_{k}+1$, and $\operatorname{Inv}(v) \backslash \operatorname{Inv}(u)=\{(i, k)\}$.

(ii) $\Pi_{\downarrow}^{J}(u)=\Pi_{\downarrow}^{J}(v)$.

(iii) $\Pi_{\uparrow}^{J}(u)=\Pi_{\uparrow}^{J}(v)$.

Proof. Let $u \lessdot_{S} v$. By definition we have $\operatorname{Inv}(v) \backslash \operatorname{Inv}(u)=\{(i, k)\}$ with $v_{i}=v_{k}+1$. Observe that $i<k$ implies that $i$ and $k$ belong to different $J$-regions.

Suppose that (i) holds. By assumption there is a $(J, 231)$-pattern in $v$, which is induced by the indices $i<j<k$. In view of Lemma 11 we conclude that $\operatorname{Inv}(v)$ is not $J$ compressed. By construction $u$ is the lower cover of $v$ which has $v_{i}$ and $v_{k}$ exchanged. The proof that $\Pi_{\downarrow}^{J}(u)=\Pi_{\downarrow}^{J}(v)$ now proceeds as in Lemma 12. This proves that (i) implies (ii). An analogous argument using Lemma 18 proves that (i) implies (iii).

Now suppose that (i) does not hold. In other words, assume that for any $j \in[n]$ which satisfies $i<j<k$ and does not belong to the same $J$-region as $i$ or $k$ we have $v_{j}<v_{i}$. 
In particular, $i$ and $k$ do not participate in any $(J, 231)$-pattern of $v$, and the maximality of $\Pi_{\downarrow}^{J}(v)$ implies that $(i, k) \in \operatorname{Inv}\left(\Pi_{\downarrow}^{J}(v)\right)$. On the other hand, since $(i, k) \notin \operatorname{Inv}(u)$ we conclude $(i, k) \notin \operatorname{Inv}\left(\Pi_{\downarrow}^{J}(u)\right)$. Hence $\Pi_{\downarrow}^{J}(u)<_{S} \Pi_{\downarrow}^{J}(v)$. This proves that (ii) implies (i). We also see that $i$ and $k$ do not participate in any $(J, 132)$-pattern of $u$, and the minimality of $\Pi_{\uparrow}^{J}(u)$ implies $(i, k) \notin \operatorname{Inv}\left(\Pi_{\uparrow}^{J}(u)\right)$. On the other hand $(i, k) \in \operatorname{Inv}(v) \subseteq \operatorname{Inv}\left(\Pi_{\uparrow}^{J}(v)\right)$. Hence $\Pi_{\uparrow}^{J}(u)<_{S} \Pi_{\uparrow}^{J}(v)$. This proves that (iii) implies (i) and the proof is complete.

Lemma 21. If $\Pi_{\downarrow}^{J}(u)=\Pi_{\downarrow}^{J}(v)$ for some $u, v \in \mathfrak{S}_{n}^{J}$, then $\Pi_{\uparrow}^{J}(u)=\Pi_{\uparrow}^{J}(v)$.

Proof. Assume that $u \leqslant_{S} v$. If $u \lessdot_{S} v$, then the claim follows from Lemma 20. If $u<_{S} v$ do not form a cover relation, we find the desired equality by repeated application of Lemma 20 using Lemma 17.

Otherwise, suppose that $u$ and $v$ are incomparable. Then, $\Pi_{\downarrow}^{J}(u \wedge v)=\Pi_{\downarrow}^{J}(u)$, since $\Pi_{\downarrow}^{J}(u)=\Pi_{\downarrow}^{J}(v)$ is the unique maximal $(J, 231)$-avoiding permutation below both $u$ and $v$. Since $u \wedge v \leqslant_{S} u$ and $u \wedge v \leqslant_{S} v$, we conclude $\Pi_{\uparrow}^{J}(u)=\Pi_{\uparrow}^{J}(u \wedge v)=\Pi_{\uparrow}^{J}(v)$ using the argument above.

Proposition 22. The equivalence relation $\Theta$ from (2) is in fact a lattice congruence on $\left[e, w_{\circ}^{J}\right]$, and the corresponding quotient lattice is $\mathcal{T}_{n}^{J}$.

Proof. Lemma 12 implies that the equivalence classes of $\Theta$ have a least element, and these minimal elements are precisely the elements of $\mathfrak{S}_{n}^{J}(231)$. Lemma 21 implies together with Lemma 18 that equivalence classes have a greatest element, and Lemma 17 implies that the equivalence class $[w]_{\Theta}$ is in fact equal to the interval $\left[\Pi_{\downarrow}^{J}(w), \Pi_{\uparrow}^{J}(w)\right]$. Lemma 19 now completes the proof.

Proof of Theorem 1. This follows from Propositions 14 and 22.

\section{Parabolic Noncrossing Partitions}

In this section, we define the set $\mathrm{NC}_{n}^{J}$ of noncrossing partitions for parabolic quotients, and give an explicit bijection between $\mathrm{NC}_{n}^{J}$ and $\mathfrak{S}_{n}^{J}(231)$.

Recall that a set partition of $[n]$ is a collection $\mathbf{P}=\left\{P_{1}, P_{2}, \ldots, P_{s}\right\}$ of pairwise disjoint, nonempty subsets of $[n]$ with the property that their union is $[n]$. The elements $P_{i}$ of $\mathbf{P}$ are called the parts of $\mathbf{P}$. A pair $(a, b)$ is a bump of $\mathbf{P}$ if $a, b \in P_{i}$ for some $i \in[s]$ and there is no $c \in P_{i}$ with $a<c<b$. Classically, a set partition is noncrossing if it does not contain two bumps $\left(i_{1}, j_{1}\right)$ and $\left(i_{2}, j_{2}\right)$ such that $i_{1}<i_{2}<j_{1}<j_{2}$ [16]. We introduce the following generalization.

Definition 23. A partition $\mathbf{P}$ of $[n]$ is J-noncrossing if it satisfies the following three conditions.

(NC1) If $i$ and $j$ lie in the same $J$-region, then they are not contained in the same part of $\mathbf{P}$. 


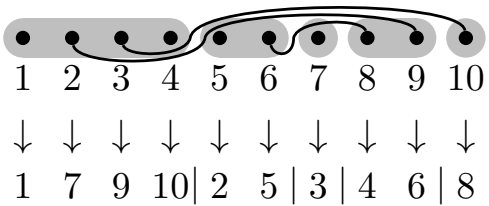

$\{7\}$

$\{5\} \quad\{6,8\}$

$\{1\}\{2,9\}\{3,10\}\{4\}$

Figure 2: The noncrossing partition $\mathbf{P}=\{\{1\},\{2,9\},\{3,10\},\{4\},\{5\},\{6,8\},\{7\}\}$ with respect to $n=10$ and $J=\left\{s_{1}, s_{2}, s_{3}, s_{5}, s_{8}\right\}$, the corresponding partially ordered set $\vec{O}_{\mathbf{P}}$, and the $(J, 231)$-avoiding permutation constructed from $\mathbf{P}$.

(NC2) If two distinct bumps $\left(i_{1}, i_{2}\right)$ and $\left(j_{1}, j_{2}\right)$ of $\mathbf{P}$ satisfy $i_{1}<j_{1}<i_{2}<j_{2}$, then either $i_{1}$ and $j_{1}$ lie in the same $J$-region or $i_{2}$ and $j_{1}$ lie in the same $J$-region.

(NC3) If two distinct bumps $\left(i_{1}, i_{2}\right)$ and $\left(j_{1}, j_{2}\right)$ of $\mathbf{P}$ satisfy $i_{1}<j_{1}<j_{2}<i_{2}$, then $i_{1}$ and $j_{1}$ lie in different $J$-regions.

We denote the set of all $J$-noncrossing set partitions of $[n]$ by $\mathrm{NC}_{n}^{J}$. If $J=\emptyset$, then we recover the classical noncrossing set partitions. We now introduce a combinatorial model for the $J$-noncrossing partitions. We draw $n$ dots, labeled by the numbers $1,2, \ldots, n$, on a straight line, and highlight the $J$-regions by grouping the corresponding dots together. For any bump $(i, j)$ in $\mathbf{P} \in \mathrm{NC}_{n}^{J}$, we draw an arc connecting the dots corresponding to $i$ and $j$, respectively, that passes below all dots corresponding to indices $k>i$ that lie in the same $J$-region as $i$, and above all other dots between $i$ and $j$. See the bottom left of Figure 4 for an illustration.

Let $\mathbf{P} \in \mathrm{NC}_{n}^{J}$ and define a binary relation $\vec{R}_{\mathbf{P}}$ on the parts of $\mathbf{P}$ by setting $\left(B, B^{\prime}\right) \in \vec{R}_{\mathbf{P}}$ if there exists a bump $\left(i_{1}, i_{2}\right)$ of $\mathbf{P}$ with $i_{1}, i_{2} \in B$ such that $i_{1}<\min B^{\prime}<i_{2}$. This relation is certainly acyclic, and can therefore be extended to an order relation by taking reflexive and transitive closures. Let $\vec{O}_{\mathbf{P}}$ be the partially ordered set whose ground sets are the parts of $\mathbf{P}$, and whose order relation is the reflexive and transitive closure of $\vec{R}_{\mathbf{P}}$.

Figure 2 illustrates this construction in the case $n=10$ and $J=\left\{s_{1}, s_{2}, s_{3}, s_{5}, s_{8}\right\}$. We may now prove the following theorem.

Theorem 24. For $n>0$ and $J \subseteq S$, there is a bijection $\mathfrak{S}_{n}^{J}(231) \simeq \mathrm{NC}_{n}^{J}$.

Proof. Let $w \in \mathfrak{S}_{n}^{J}(231)$. We construct a set partition $\mathbf{P}$ of $[n]$ by associating a bump $(i, j)$ with every descent $(i, j) \in \operatorname{Des}(w)$. If $(i, j)$ is a bump of $\mathbf{P}$, then $(i, j) \in \operatorname{Des}(w)$, and Lemma 5 implies that $i$ and $j$ lie in different $J$-regions. This establishes condition (NC1). Suppose $\left(i_{1}, i_{2}\right)$ and $\left(j_{1}, j_{2}\right)$ are two different bumps of $\mathbf{P}$ with $i_{1}<j_{1}<i_{2}<j_{2}$, but neither $i_{1}, j_{1}$ nor $i_{2}, j_{1}$ are in the same $J$-region. If $w_{i_{1}}<w_{j_{1}}$, then $\left(i_{1}, j_{1}, i_{2}\right)$ is a $(J, 231)$-pattern in $w$, which is a contradiction. If $w_{i_{1}}>w_{j_{1}}$, it follows that $w_{j_{1}}<w_{i_{2}}$, and then $\left(j_{1}, i_{2}, j_{2}\right)$ is a $(J, 231)$-pattern in $w$, which is a contradiction. Hence (NC2) is satisfied. Finally, suppose that $\left(i_{1}, i_{2}\right)$ and $\left(j_{1}, j_{2}\right)$ are two different bumps of $\mathbf{P}$ with $i_{1}<j_{1}<j_{2}<i_{2}$ such that $i_{1}$ and $j_{1}$ are in the same $J$-region. Lemma 5 implies $w_{i_{1}}<w_{j_{1}}$. 
It follows that $\left(i_{1}, j_{1}, i_{2}\right)$ is a $(J, 231)$-pattern in $w$, which is a contradiction. Hence (NC3) is satisfied, and so $\mathbf{P} \in \mathrm{NC}_{n}^{J}$.

Conversely, let $\mathbf{P} \in \mathrm{NC}_{n}^{J}$. We construct a permutation $w \in \mathfrak{S}_{n}^{J}(231)$ where every bump $(i, j)$ of $\mathbf{P}$ corresponds to a descent $(i, j) \in \operatorname{Des}(w)$. We proceed by induction on $n$, with the case $n=1$ being trivial. Suppose that for any $n^{\prime}<n$ we can construct a $\left(J^{\prime}, 231\right)$-avoiding permutation of $\mathfrak{S}_{n^{\prime}}^{J^{\prime}}$ from a given $J^{\prime}$-noncrossing set partition of $\left[n^{\prime}\right]$, where $J^{\prime}$ is the restriction of $J$ to $\left[n^{\prime}\right]$.

Let $\vec{O}_{\mathbf{P}}$ be the partially ordered set on the parts of $\mathbf{P}$ that we have defined just before this theorem. Let $\bar{P}$ be the unique part of $\mathbf{P}$ containing 1 , and let $X$ be the set of all integers that belong to parts in the order filter generated by $\bar{P}$. We set $w(1)=|X|$.

If we remove $\bar{P}$ from $\mathbf{P}$, then we obtain two smaller partitions from the remaining parts. The elements in $X \backslash \bar{P}$ form a left partition $\mathbf{P}_{l}$, and the elements in $[n] \backslash X$ form a right partition $\mathbf{P}_{r}$. Both $\mathbf{P}_{l}$ and $\mathbf{P}_{r}$ can be seen as parabolic noncrossing set partitions of $\left[n_{l}\right]$ and $\left[n_{r}\right]$, respectively, where $n_{l}, n_{r}<n$. By induction we can create $(J, 231)$-avoiding permutations $w^{(l)}$ and $w^{(r)}$ from these partitions (and we may reuse $\vec{O}_{\mathbf{P}}$ for that). Now we obtain the value $w_{j}$ for $j \notin \bar{P}$ as follows. If $j \in \mathbf{P}_{l}$, then $w_{j}=w_{j^{\prime}}^{(l)}$ if $j$ is the $\left(j^{\prime}\right)^{\text {th }}$ largest value in $\mathbf{P}_{l}$. If $j \in \mathbf{P}_{r}$, then $w_{j}=w_{j^{\prime}}^{(r)}+|X|$ if $j$ is the $\left(j^{\prime}\right)^{\text {th }}$ largest value in $\mathbf{P}_{r}$.

Since all bumps in $\mathbf{P}$ occur only between elements in $\bar{P}$, in $\mathbf{P}_{l}$, or in $\mathbf{P}_{r}$, it follows that $w \in \mathfrak{S}_{n}^{J}(231)$.

Example 25. Let $J=\left\{s_{1}, s_{2}, s_{3}, s_{5}, s_{8}\right\}$. Consider $\mathbf{P} \in \mathrm{NC}_{10}^{J}$ given by the bumps $(2,9),(3,10),(6,8)$. This partition is displayed in the top-left part of Figure 2, the corresponding poset $\vec{O}_{\mathbf{P}}$ on the right. Since no bump starts in 1 , we obtain $w_{1}=1$, and the corresponding right partition is the restriction of $\mathbf{P}$ to $\{2,3, \ldots, 10\}$. Here we have $\bar{P}=\{2,9\}$, and we have $X=\{2,5,6,7,8,9\}$. Hence we obtain $w_{2}=7$ and $w_{9}=6$. The corresponding left partition is $\mathbf{P}_{l}=\{\{5\},\{6,8\},\{7\}\}$ and the corresponding right partition is $\mathbf{P}_{r}=\{\{3,10\},\{4\}\}$. By induction, we conclude that $w^{(l)}=14|2| 3$ and $w^{(r)}=23 \mid 1$. We fashion them together to form the permutation $w=17910|25| 3|46| 8$, which is indeed contained in $\mathfrak{S}_{10}^{J}(231)$. By construction, $\{(2,9),(3,10),(6,8)\}$ are the descents of $w$, and are precisely the bumps of $\mathbf{P}$.

Remark 26. When restricted to the $(J, 231)$-sortable elements, one can check that the bijection of Theorem 24 is identical to the bijection given in [27] between elements of the symmetric group and certain noncrossing arc diagrams. Theorem 24 was discovered independently, and appeared in [32].

\section{Parabolic Nonnesting Partitions}

The nonnesting set partitions are a second important subset of the set partitions of $[n]$. Nonnesting set partitions are characterized as not containing two bumps $\left(i_{1}, i_{2}\right)$ and $\left(j_{1}, j_{2}\right)$ such that $i_{1}<j_{1}<j_{2}<i_{2}$. These were introduced by A. Postnikov uniformly for all crystallographic Coxeter groups as order ideals in the corresponding root poset $[28$, Remark 2]. It turns out that (for any crystallographic Coxeter group) noncrossing and 
nonnesting partitions are equinumerous. Moreover, they are also equidistributed by part size [3]. We introduce the following generalization.

Definition 27. A partition $\mathbf{P}$ of $[n]$ is J-nonnesting if it satisfies the following two conditions.

(NN1) If $i$ and $j$ lie in the same $J$-region, then they are not contained in the same part of $\mathbf{P}$.

(NN2) If $\left(i_{1}, i_{2}\right)$ and $\left(j_{1}, j_{2}\right)$ are two distinct bumps of $\mathbf{P}$, then it is not the case that $i_{1}<j_{1}<j_{2}<i_{2}$.

We denote the set of all $J$-nonnesting partitions of $[n]$ by $\mathrm{NN}_{n}^{J}$. If $J=\emptyset$, then we recover the classical nonnesting set partitions.

Recall that the root poset of $\mathfrak{S}_{n}$ is the poset $\Phi_{+}=(T, \leqslant)$, where $T$ is the set of all transpositions of $\mathfrak{S}_{n}$, and we have $\left(i_{1}, i_{2}\right) \leqslant\left(j_{1}, j_{2}\right)$ if and only if $i_{1} \geqslant j_{1}$ and $i_{2} \leqslant j_{2}$. The parabolic root poset of $\mathfrak{S}_{n}$, denoted by $\Phi_{+}^{J}$, is the order filter of $\Phi_{+}$generated by the adjacent transpositions not in $J$.

We first observe that $J$-nonnesting partitions of $[n]$ are in bijection with order ideals in this parabolic root poset. See Figure 3 for an illustration.

Lemma 28. For $n>0$ and $J \subseteq S$, there is a bijection from $J$-nonnesting partitions to order ideals in $\Phi_{+}^{J}$.

Proof. Let $I$ be an order ideal of $\Phi_{+}^{J}$, and let $M$ denote the set of minimal elements in the complement of $I$. In particular, $M$ is an antichain, i.e. no two elements of $M$ are comparable. Thus if $\left(i_{1}, i_{2}\right),\left(j_{1}, j_{2}\right) \in M$ and $i_{1}<j_{1}$, then $i_{2} \in\left\{j_{1}, j_{1}+1, \ldots, j_{2}-1\right\}$ (and accordingly if $j_{1}<i_{1}$ ). Hence, (NN2) is satisfied. If there are two distinct elements $i_{0}$ and $i_{k}$ which belong to the same $J$-region and to the same part $B$ of $\mathbf{P}$, then there must be a sequence of bumps $\left(i_{0}, i_{1}\right),\left(i_{1}, i_{2}\right), \ldots,\left(i_{k-1}, i_{k}\right)$, which belong to this $J$-region and to $B$ as well. This contradicts the definition of $\Phi_{+}^{J}$, because we have specifically excluded pairs of the form $(a, b)$ with $a$ and $b$ both belonging to the same $J$-region. This contradiction shows that (NN1) is satisfied.

Conversely, let $\mathbf{P} \in \mathrm{NN}_{n}^{J}$, and let $M$ be the set of bumps of $\mathbf{P}$. By (NN1) we see that $M \subseteq \Phi_{+}^{J}$. If there exist $\left(i_{1}, i_{2}\right),\left(j_{1}, j_{2}\right) \in M$ which are comparable in $\Phi_{+}^{J}$, then without loss of generality we may assume that $i_{1} \geqslant j_{1}$ and $i_{2} \leqslant j_{2}$. Since naturally $i_{1}<i_{2}$ we obtain a contradiction to (NN2).

We now prove that $J$-nonnesting and $J$-noncrossing partitions are also in bijection. See Figure 4 for an example.

Theorem 29. For $n>0$ and $J \subseteq S$, there is a bijection $\mathrm{NN}_{n}^{J} \simeq \mathrm{NC}_{n}^{J}$.

Proof. We begin with the construction of a bijection from $\mathrm{NN}_{n}^{J}$ to $\mathrm{NC}_{n}^{J}$ for the case of maximal parabolic quotients, i.e. where $J=S \backslash\left\{s_{k}\right\}$ for $k \in[n]$. We label the transposition 


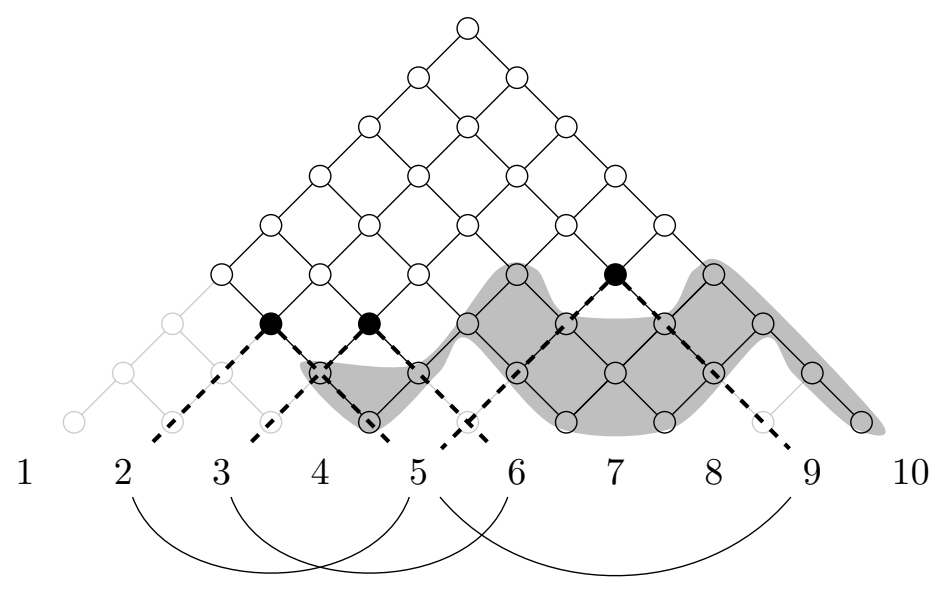

Figure 3: The parabolic root poset of $\mathfrak{S}_{10}$ with respect to $J=\left\{s_{1}, s_{2}, s_{3}, s_{5}, s_{8}\right\}$. The shaded region is an order ideal, and the minimal elements of the complement are marked in black. The corresponding $J$-nonnesting partition is $\{\{1\},\{2,5,9\},\{3,6\},\{4\},\{7\},\{8\},\{10\}\}$.

$(i, j)$ in $\Phi_{+}^{J}$ by the arc $(k+1-i, n+1-j+k)$, which yields the following labeling of $\Phi_{+}^{J}$ (under a suitable rotation):

$$
\begin{array}{cccc}
(k,(k+1)) & \cdots & (k,(n-1)) & (k, n) \\
\vdots & \vdots & \vdots & \vdots \\
(2,(k+1)) & \cdots & (2,(n-1)) & (2, n) \\
(1,(k+1)) & \cdots & (1,(n-1)) & (1, n)
\end{array}
$$

The $J$-nonnesting set partition corresponding to an order ideal in $\Phi_{+}^{J}$ is the one whose bumps are the labels of the minimal elements not in the order ideal. Since $B(J)=$ $\{\{1,2, \ldots, k\},\{k+1, k+2, \ldots, n\}\}$, condition (NC3) ensures that every $J$-noncrossing partition is also $J$-nonnesting and vice versa.

Now suppose that $J=S \backslash\left\{s_{k_{1}}, s_{k_{2}}, \ldots, s_{k_{r}}\right\}$, and let $I$ be an order ideal of $\Phi_{+}^{J}$. We construct a noncrossing partition $\mathbf{P} \in \mathrm{NC}_{n}^{J}$ inductively starting from the partition with no parts. First we break $I$ in two pieces, $A$ and $B$ : $A$ contains all the transpositions in $I$ that lie above $s_{k_{1}}$ in $\Phi_{+}^{J}$, and $B$ contains all the other transpositions in $I$. Then $B$ is an order ideal in $\Phi_{+}^{J \backslash\left\{s_{k_{1}}\right\}}$, and we can construct a $\left(J \backslash\left\{s_{k_{1}}\right\}\right)$-noncrossing set partition of $\left\{k_{1}+1, k_{1}+2, \ldots, n\right\}$ by induction. Now we choose all those columns in piece $A$ that either lie outside the order filter generated by $s_{k_{2}}, \ldots, s_{k_{r}}$ or that have an element of $I$ in piece $B$ directly below them. (We thus pick the columns of $A$ that are "supported" by B.)

Let $l_{1}, l_{2}, \ldots, l_{r}$ denote the column labels from the inductive step of the part of $B$ that supports $A$. Any bump starting in $\left\{1,2, \ldots, k_{1}\right\}$ can end either in $\left\{k_{1}+1, k_{1}+2, \ldots, k_{2}\right\}$ or in $\left\{l_{1}, l_{2}, \ldots, l_{r}\right\}$, in order not to cross any existing bumps. We label the transpositions 


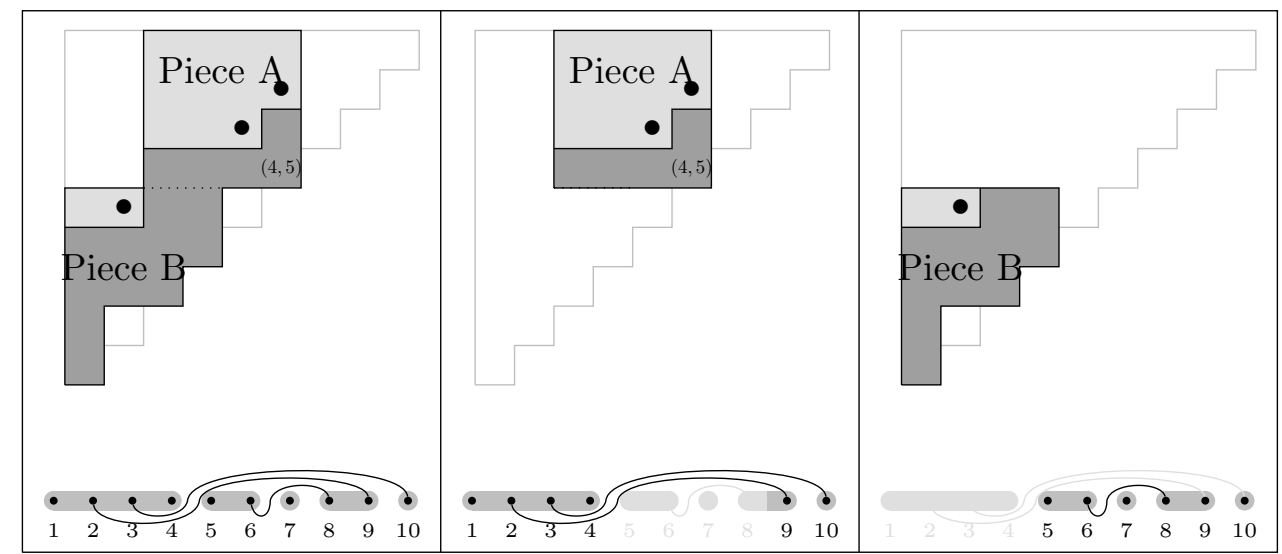

Figure 4: Figure illustrating how to combine pieces A and B.

in the chosen part of $A$ as follows:

$$
\begin{array}{cccccccc}
\left(k_{1},\left(k_{1}+1\right)\right) & \left(k_{1},\left(k_{1}+2\right)\right) & \cdots & \left(k_{1}, k_{2}\right) & \left(k, l_{1}\right) & \left(k, l_{2}\right) & \cdots & \left(k, l_{r}\right) \\
\vdots & \vdots & \vdots & \vdots & \vdots & \vdots & \vdots & \vdots \\
\left(2,\left(k_{1}+1\right)\right) & \left(2,\left(k_{1}+2\right)\right) & \cdots & \left(2, k_{2}\right) & \left(2, l_{1}\right) & \left(2, l_{2}\right) & \cdots & \left(2, l_{r}\right) \\
\left(1,\left(k_{1}+1\right)\right) & \left(1,\left(k_{1}+2\right)\right) & \cdots & \left(1, k_{2}\right) & \left(1, l_{1}\right) & \left(1, l_{2}\right) & \cdots & \left(1, l_{r}\right)
\end{array}
$$

The labels corresponding to the minimal transpositions that are not in $I$ within these chosen columns then yield the remaining bumps. By construction, the resulting partition is $J$-noncrossing.

The inverse map is constructed by temporarily forgetting about the bumps from the first $J$-region, and then using the smaller $J$-noncrossing partition to construct the $B$ piece of the order ideal inductively. From there, we can again identify the "supported" columns in the $A$ piece, and the bumps starting in the first $J$-region then give the remaining elements of the order ideal.

Example 30. Consider the $J$-nonnesting set partition of [10] shown at the top left of Figure 4 indicated by the dark gray region. The construction of the smaller parabolic noncrossing partitions is shown in the middle and right part of that figure, and the resulting $J$-noncrossing partition is shown at the bottom left.

Proof of Theorem 2. This follows from Theorems 24 and 29.

Recall that a Ferrers shape is a sequence $\lambda=\left(\lambda_{1}, \lambda_{2}, \ldots, \lambda_{k}\right)$ of positive integers with $\lambda_{1} \geqslant \lambda_{2} \geqslant \cdots \geqslant \lambda_{k}$. Another Ferrers shape $\left(\lambda_{1}^{\prime}, \lambda_{2}^{\prime}, \ldots, \lambda_{k}^{\prime}\right)$ fits inside $\lambda$ if $\lambda_{i}^{\prime} \leqslant \lambda_{i}$ for all $i \in[k]$.

The parabolic root poset — when rotated by 45 degrees counterclockwise — can be viewed as a bounding Ferrers shape

$$
\lambda_{n}^{J}:=\left(j_{r}^{n-j_{r}}, \ldots, j_{2}^{j_{3}-j_{2}}, j_{1}^{j_{2}-j_{1}}\right)
$$


and the complements of the order ideals in this poset correspond precisely to the Ferrers shapes that fit inside $\lambda_{n}^{J}$. In our running example we obtain $\lambda_{10}^{\left\{s_{1}, s_{2}, s_{3}, s_{5}, s_{8}\right\}}=(9,7,7,6,4,4)$ as can quickly be verified in Figure 3. The enumeration of Ferrers shapes fitting into $\lambda_{n}^{J}$ now follows from the next result due to G. Kreweras, and allows for computing the parabolic Catalan numbers.

Theorem 31 ([15, Section 2.3.7]). If $\lambda=\left(\lambda_{1}, \lambda_{2}, \ldots, \lambda_{k}\right)$ is a Ferrers shape, then the number of Ferrers shapes that fits inside $\lambda$ is given by the determinant of the $k \times k$-matrix whose entry in row $i$ and column $j$ is $\left(\begin{array}{c}\lambda_{j}+1 \\ j-i+1\end{array}\right)$.

\section{Generalization to Coxeter Groups}

In recent years, 231-avoiding permutations, noncrossing set partitions, and nonnesting set partitions have each been generalized from the symmetric group to finite Coxeter groups - see [25], [4,8], and [28, Remark 2], respectively. These generalizations allow for further parametrization by a Coxeter element (a product of the simple reflections in some order). In this section, we describe a generalization of our parabolic versions of these objects in a similar fashion.

\subsection{Coxeter Groups}

We first recall some background on Coxeter groups. For more details see [5,13]. A Coxeter system is a pair $(W, S)$, where $W$ is a group and $S=\left\{s_{1}, s_{2}, \ldots, s_{n}\right\} \subseteq W$ is a generating set such that $W$ admits the presentation

$$
\left.W=\left\langle s_{1}, s_{2}, \ldots, s_{n}\right|\left(s_{i} s_{j}\right)^{m_{i, j}}=e \text { for } i, j \in[n]\right\rangle,
$$

where $e$ denotes the identity of $W$. The parameters $m_{i, j}$ are positive integers or the formal symbol $\infty$, where $m_{i, j}=1$ if and only if $i=j$. If $m_{i, j}=\infty$, then there is no relation between the generators $s_{i}$ and $s_{j}$. In this situation we call $W$ a Coxeter group and the cardinality $n=|S|$ the rank of $W$. For geometric reasons we call the elements of $S$ the simple reflections, and define the set of all reflections by $T=\left\{w s w^{-1} \mid w \in W, s \in S\right\}$.

Since $S$ generates $W$, every $w \in W$ can be written as a product of the elements in $S$. A reduced expression for $w$ is such a product of minimal length, and this length is called the Coxeter length of $w$; denoted by $\ell_{S}(w)$. The (right) weak order on $W$ is the partial order $\leqslant_{S}$ defined by $u \leqslant_{S} v$ if and only if $\ell_{S}(v)=\ell_{S}(u)+\ell_{S}\left(u^{-1} v\right)$. We write Weak $(W)$ for the partially ordered set $\left(W, \leqslant_{S}\right)$.

Remark 32. In Section 2.1 we defined a left weak order on the symmetric group $\mathfrak{S}_{n}$, which may be generalized to Coxeter groups via the condition $\ell_{S}(v)=\ell_{S}(u)+\ell_{S}\left(v u^{-1}\right)$. The map $w \mapsto w^{-1}$ is a poset isomorphism from left to right weak order, so that the results from Section 3 could be phrased equally well in terms of right weak order.

In general, $\operatorname{Weak}(W)$ is a meet-semilattice - if $W$ is finite, then Weak $(W)$ has a unique longest element $w_{\mathrm{o}}$, which implies that $\operatorname{Weak}(W)$ is in fact a lattice [5, Theorem 3.2.1]. 
Any $J \subseteq S$ naturally generates a (parabolic) subgroup $W_{J}$ of $W$, and the set of minimal length representatives of the right cosets of $W$ by $W_{J}$ forms the parabolic quotient $W^{J}$ of $W$ with respect to $J$. Proposition 2.4.4 in [5] implies that any $w \in W$ can be factorized uniquely as $w=w^{J} \cdot w_{J}$, where $w^{J} \in W^{J}$ and $w_{J} \in W_{J}$. The weak order on $W$ gives a partial order on $W^{J}$, and Weak $\left(W^{J}\right)$ is isomorphic to the weak order ideal $\left[e, w_{\circ}^{J}\right]$ when $W$ is finite [6, Theorem 4.1].

Comparing the presentations (1) and (3), we see that the symmetric group with the generating set of all adjacent transpositions forms a Coxeter system, and the reflections are all conjugates of the adjacent transpositions. We use this correspondence to generalize the notion of an inversion from the symmetric group to all Coxeter groups. A (left) inversion of $W$ is a reflection $t \in T$ such that $\ell_{S}(t w)<\ell_{S}(w)$. The set of all inversions of $w$ is denoted by $\operatorname{Inv}(w)$. Analogously to the symmetric group, we can give an equivalent definition of the weak order by setting $u \leqslant_{S} v$ if and only if $\operatorname{Inv}(u) \subseteq \operatorname{Inv}(v)$ [5, Proposition 3.1.3].

We want to emphasize a special subset of the inversions. A cover reflection of $w$ is an inversion $t \in \operatorname{Inv}(w)$ such that there exists some $s \in S$ with $t w=w s$. The name comes from the fact that multiplying some element by a simple reflection produces a cover relation in the weak order, and the cover reflection is then the conjugate of this simple reflection by the larger element in this cover. The set of cover reflections of $w$ is denoted by $\operatorname{Cov}(w)$.

Now fix a reduced expression $\mathbf{w}=a_{1} a_{2} \cdots a_{k}$. The inversion sequence of $\mathbf{w}$ is the sequence $r_{1}, r_{2}, \ldots, r_{k}$, where $r_{i}=a_{1} a_{2} \cdots a_{i-1} a_{i} a_{i-1} \cdots a_{2} a_{1}$. It is clear by construction that $\operatorname{Inv}(w)=\left\{r_{1}, r_{2}, \ldots, r_{k}\right\}$. Observe, however, that the inversion sequence equips $\operatorname{Inv}(w)$ with a linear order; the inversion order $r_{1}<r_{2}<\cdots<r_{k}$. This order will be denoted by $\operatorname{Inv}(\mathbf{w})$.

Since the elements of $T$ geometrically act as reflections on a Euclidean vector space, we can associate two normal vectors to the corresponding reflecting hyperplane. The collection of all these normal vectors is a root system of $W$, and it can be partitioned into positive and negative roots. It follows that there is a bijection from $T$ to the set $\Phi^{+}$of all positive roots. Given $\alpha \in \Phi^{+}$, let $t_{\alpha} \in T$ be the corresponding reflection. It follows from [10, Lemma 4.1(iv)] that whenever we have a reflection $t_{a \alpha+b \beta} \in \operatorname{Inv}(w)$ for some $\alpha, \beta \in \Phi^{+}$and some positive integers $a, b$, then at least one of $t_{\alpha}$ and $t_{\beta}$ are in $\operatorname{Inv}(w)$ as well. We refer the interested reader to [13] for more background on the geometric realization of Coxeter groups.

Finally, we recall the existence of some special, well-behaved reduced expressions for any element of $W$. A Coxeter element of $(W, S)$ is an element that has a reduced expression which is a permutation of all simple reflections. Fix such a Coxeter element $c \in W$. Clearly, any reduced expression of $w \in W$ appears as a subword of the half-infinite word $c^{\infty}$ (which is the infinite concatenation of a fixed reduced expression for $c$ ). The $c$-sorting word of $w$ is the reduced expression for $w$ which appears leftmost in $c^{\infty}$, and will be denoted by $\mathbf{w}(\mathbf{c})$. 


\subsection{Aligned Elements for Parabolic Quotients}

In [24, Section 4], N. Reading defined a notion of $c$-alignment for the elements of a finite Coxeter group $W$ with respect to some Coxeter element $c \in W$. More precisely, an element $w$ of $W$ is c-aligned if whenever we have $t_{\alpha}<t_{a \alpha+b \beta}<t_{\beta}$ in the inversion order $\operatorname{Inv}\left(\mathbf{w}_{\circ}(\mathbf{c})\right)$, where $\alpha, \beta \in \Phi^{+}$and $a, b$ are positive integers, then $t_{a \alpha+b \beta} \in \operatorname{Inv}(w)$ implies $t_{\alpha} \in \operatorname{Inv}(w)$. For $W=\mathfrak{S}_{n}$ and $c=s_{n-1} \cdots s_{2} s_{1}$ the linear Coxeter element, the $c$-aligned elements are precisely the 231-avoiding permutations.

We now propose a definition of $c$-aligned elements for parabolic quotients.

Definition 33. Let $(W, S)$ be a finite Coxeter system, let $J \subseteq S$, and let $c \in W$ be a Coxeter element. An element $w \in W^{J}$ is $\left(W^{J}, c\right)$-aligned if, whenever we have $t_{\alpha}<$ $t_{a \alpha+b \beta}<t_{\beta}$ in $\operatorname{Inv}\left(\mathbf{w}_{\circ}^{\mathbf{J}}(\mathbf{c})\right)$, where $\alpha, \beta \in \Phi^{+}$and $a, b$ are positive integers, then $t_{a \alpha+b \beta} \in$ $\operatorname{Cov}(w)$ implies $t_{\alpha} \in \operatorname{Inv}(w)$.

We denote the set of all $\left(W^{J}, c\right)$-aligned elements of $W$ by $\operatorname{Align}\left(W^{J}, c\right)$.

There is a subtlety in Definition 33-we only require the root $t_{a \alpha+b \beta}$ to correspond to a cover reflection of $w$, rather than to an arbitrary inversion. It was shown in [23, Lemma 5.5] and follows from [24, Lemmas 4.9 and 4.11] that our parabolic aligned condition for $J=\emptyset$ is indeed equivalent to the original aligned condition for Coxeter groups of type $A, B$, and $D$. This equivalence is trivial for the dihedral groups, and it was checked by computer for the groups $H_{3}, H_{4}$, and $F_{4}$. The remaining exceptional groups $E_{6}, E_{7}$, and $E_{8}$ have not been checked by computer. See also [32, Remark 5.1.8].

The following lemma states that our parabolic pattern avoidance condition from Definition 6 is equivalent to Definition 33 in the case of the symmetric group and the linear Coxeter element $c=s_{1} s_{2} \cdots s_{n-1}$, where $s_{i}=(i, i+1)$.

Lemma 34. Let $W=\mathfrak{S}_{n}, c=s_{1} s_{2} \cdots s_{n-1}$, and choose $J \subseteq S$. An element $w \in \mathfrak{S}_{n}^{J}$ is $\left(W^{J}, c\right)$-aligned if and only if $w^{-1}$ is $(J, 231)$-avoiding.

Proof. By definition, cover reflections of $w \in \mathfrak{S}_{n}$ correspond to descents of $w^{-1}$ so that Definition 33 agrees with Definition 10 after taking inverses. Lemma 11 then implies that $w \in \mathfrak{S}_{n}^{J}$ is $\left(W^{J}, c\right)$-aligned if and only if $w^{-1}$ is $(J, 231)$-avoiding.

It is an intriguing question whether the statement of Theorem 1 survives this generalization, and computer experiments have led us to formulate the following conjecture.

Conjecture 35. For any finite Coxeter system $(W, S)$, any $J \subseteq S$, and any Coxeter element $c \in W$, the poset $\operatorname{Weak}\left(\operatorname{Align}\left(W^{J}, c\right)\right)$ is a lattice. Moreover, it is a lattice quotient of $\operatorname{Weak}\left(W^{J}\right)$.

Conjecture 35 holds in two interesting cases - when $J=\emptyset[25$, Theorem 1.1], and when $J=S \backslash\{s\}$ is chosen such that $w_{\circ}^{J}$ is fully commutative, i.e. any two reduced expressions for $w_{\circ}^{J}$ differ only by commutations. In the latter case, we simply have $\operatorname{Align}\left(W^{J}, c\right)=W^{J}$ for any $c$, and the lattice property follows from the fact that $\left(W^{J}, \leqslant_{S}\right)$ is an interval in the weak order on $W$ [32, Section 5.2]. See [30] for more background on fully commutative elements, and a characterization of the sets $J=S \backslash\{s\}$ such that $w_{\circ}^{J}$ is fully commutative. The remaining cases, however, are wide open. 


\subsection{Noncrossing Partitions for Parabolic Quotients}

Let us continue with the generalization of noncrossing set partitions to parabolic quotients of finite Coxeter groups. Recall that the original definition of noncrossing partitions associated with a pair $(W, c)$ is in terms of elements below $c$ in a certain partial order depending on all reflections of $W[4,8]$. It was observed by N. Reading in [24, Theorem 6.1] that the $(W, c)$-noncrossing partitions are determined bijectively by the cover reflections of the $c$-aligned elements of $W$. The next definition is a straightforward generalization of this correspondence. For $w \in W^{J}$, and suppose that $\operatorname{Cov}(w)=\left\{r_{1}, r_{2}, \ldots, r_{k}\right\}$, where the order of the cover reflections comes from $\operatorname{Inv}\left(\mathbf{w}_{\mathbf{o}}^{\mathbf{J}}(\mathbf{c})\right)$. Let $\psi(w)=r_{1} r_{2} \cdots r_{k}$.

Definition 36. Let $(W, S)$ be a finite Coxeter system, let $J \subseteq S$, and let $c \in W$ be a Coxeter element. The $\left(W^{J}, c\right)$-noncrossing partitions are the elements in the image of $\psi$ restricted to $\operatorname{Align}\left(W^{J}, c\right)$.

We denote the set of $\left(W^{J}, c\right)$-noncrossing partitions by $\mathrm{NC}\left(W^{J}, c\right)$. It follows from Theorem 24 that this definition coincides with Definition 23 when we consider the symmetric group and the linear Coxeter element.

\subsection{Nonnesting Partitions for Parabolic Quotients}

We now generalize the nonnesting set partitions to parabolic quotients of finite Coxeter groups. In the classical setting, nonnesting partitions are defined as follows for any finite irreducible Coxeter group that is not isomorphic to $H_{4}$. If $W$ is an irreducible crystallographic Coxeter group, i.e. we have $m_{i j} \in\{1,2,3,4,6\}$ in (3), then we can partially order the positive roots of $W$ by $\alpha \leqslant \beta$ if and only if $\beta-\alpha$ can be expressed as a linear combination of the simple roots with only positive coefficients. This partial order yields the root poset of $W$. Root posets for the remaining finite irreducible Coxeter groups other than $H_{4}$ were suggested in [1, Figure 5.15]. The $W$-nonnesting partitions of $W$ are then the order ideals in the root poset. In particular, they do not depend on a Coxeter element. Recall that the parabolic root poset of $W$ with respect to $J$ is defined to be the order filter in the root poset of $W$ induced by the simple reflections not in $J$.

Definition 37. Let $(W, S)$ be a finite Coxeter system, with $W \neq H_{4}$, and let $J \subseteq S$. The $W^{J}$-nonnesting partitions are the order ideals in the parabolic root poset of $W$ with respect to $J$.

We denote the set of $W^{J}$-nonnesting partitions by $\mathrm{NN}\left(W^{J}\right)$. It is clear that this definition coincides with Definition 27 when we consider the symmetric group.

\subsection{Subword Complexes for Parabolic Quotients}

However, this is not the end of the story. There is yet another family of combinatorial objects that seems to fit nicely into the presented framework. Let $(W, S)$ be a finite Coxeter system, let $Q$ be a word on the alphabet $S$, and let $w \in W$. The subword complex $\mathrm{SW}(Q, w)$ is the pure simplicial complex whose facets are the subwords $Q-P$ such that 
$P$ is a reduced expression for $w$ [14]. For our purpose, the following subword complex shall be emphasized.

Definition 38. Let $(W, S)$ be a finite Coxeter system, let $J \subseteq S$, and let $c \in W$ be a Coxeter element. The $\left(W^{J}, c\right)$-cluster complex is the subword complex $\mathrm{SW}\left(\mathbf{c w}_{\circ}(\mathbf{c}), w_{\circ}^{J}\right)$.

We denote the $\left(W^{J}, c\right)$-cluster complex by $\mathrm{SW}\left(W^{J}, c\right)$, and denote its number of facets by $\left|\mathrm{SW}\left(W^{J}, c\right)\right|$. The next result states that $\left|\mathrm{SW}\left(\mathfrak{S}_{n}^{J}, c\right)\right|$, where $c$ is the linear Coxeter element, equals the number appearing (implicitly) in Theorem 2.

Proposition 39. Let $n>0$, let $S$ be the set of adjacent transpositions, and let $c$ be the linear Coxeter element. For $J \subseteq S$, we have $\left|\mathrm{SW}\left(\mathfrak{S}^{J}, c\right)\right|=\left|\mathrm{NN}_{n}^{J}\right|$.

Proof. Recall from Section 5 that the elements in $\mathrm{NN}_{n}^{J}$ are order ideals in the parabolic root poset of $\mathfrak{S}_{n}^{J}$, and this poset can be interpreted as the Ferrers shape $\lambda_{n}^{J}$.

For any Ferrers shape $\lambda=\left(\lambda_{1}, \lambda_{2}, \ldots, \lambda_{t}\right)$ we may consider the permutation

$$
w(\lambda):=\prod_{i=1}^{t} \prod_{j=\lambda_{1}+2-i-\lambda_{i}}^{\lambda_{1}+1-i} s_{j} .
$$

It is straightforward to verify that in the case where $\lambda=\lambda_{n}^{J}$ describes the shape of the parabolic root poset of $\mathfrak{S}_{n}^{J}$ the element $w(\lambda)$ is precisely $w_{\circ}^{J}$. The result follows then from [29, Theorem 1.1]. See also [32, Remark 4.5.8].

Since $\mathrm{SW}\left(W^{J}, c\right)$ is a subword complex, there is a natural poset structure on its facets. More generally, let $F, F^{\prime}$ be two facets of a subword complex $\operatorname{SW}(Q, w)$ such that $F-\{i\}=F^{\prime}-\{j\}$ for some $i \in F$, and some $j \in F^{\prime}$. If $i<j$, then we call $F \rightarrow F^{\prime}$ a flip, and the facets of $\mathrm{SW}(Q, w)$ together with the set of flips forms an acyclic graph, and therefore its reflexive and transitive closure is the flip poset of $\operatorname{SW}(Q, w)[14$, Remark 4.5].

Conjecture 40. For any finite Coxeter system $(W, S)$, any $J \subseteq S$, and any Coxeter element $c \in W$, the restriction of the weak order to $\left(W^{J}, c\right)$-aligned elements is isomorphic to the flip poset of $\mathrm{SW}\left(W^{J}, c\right)$.

\subsection{Numerology}

In this section we describe how the objects defined in Sections 6.2 to 6.5 conjecturally fit together from an enumerative point of view. It is well known that for any finite Coxeter group $W$ and any Coxeter element $c \in W$ we have

$$
\left|\operatorname{Align}\left(W^{\emptyset}, c\right)\right|=\left|\mathrm{NC}\left(W^{\emptyset}, c\right)\right|=\left|\mathrm{NN}\left(W^{\emptyset}\right)\right|=\left|\mathrm{SW}\left(W^{\emptyset}, c\right)\right|,
$$

see for instance [25, Theorem 6.1] and [2], and this cardinality is given by the well-known $W$-Catalan number [28, Remark 2]. We have shown in Theorem 2 and Proposition 39 that this statement can be generalized to parabolic quotients of the symmetric group and the linear Coxeter element. It turns out, however, that this statement does not hold in 
general for any parabolic quotient of any Coxeter group and any Coxeter element. Take for instance $W=D_{4}, J=\left\{s_{1}, s_{2}\right\}$, and $c=s_{3} s_{2} s_{1} s_{4}$, where $s_{2}$ is the simple reflection that does not commute with any of the other simple reflections. In this case we have $\left|\operatorname{Align}\left(W^{J}, c\right)\right|=21$, but $\left|\mathrm{NN}\left(W^{J}\right)\right|=22$.

Another related question is, whether the cardinality of the sets $\operatorname{Align}\left(W^{J}, c\right)$ and $\mathrm{NC}\left(W^{J}, c\right)$ is independent of the choice of $c$. This property is known for $J=\emptyset$, see for instance [24, Theorem 9.1], but it turns out once more that it does not hold for any parabolic quotient of any Coxeter group and any Coxeter element. Take again $W=D_{4}$, $J=\left\{s_{1}, s_{2}\right\}, c=s_{3} s_{2} s_{1} s_{4}$ as above and take $c^{\prime}=s_{2} s_{3} s_{4} s_{1}$. We then have $\left|\operatorname{Align}\left(W^{J}, c\right)\right|=$ 21 and $\left|\operatorname{Align}\left(W^{J}, c^{\prime}\right)\right|=22$.

As a consequence we conclude that, in general, there is no well-defined parabolic Coxeter-Catalan number $\operatorname{Cat}\left(W^{J}\right)$. See Figures 1 to 5 for more data.

\begin{tabular}{|c|c|c|c|c|c|c|}
\hline$J$ & $\operatorname{Align}\left(A_{4}^{J}\right)$ & $\begin{array}{l}= \\
= \\
=\end{array}$ & $\begin{array}{l}\mathrm{NC}\left(A_{4}^{J}\right) \\
\operatorname{SW}\left(A_{4}^{J}\right) \\
\operatorname{NN}\left(A_{4}^{J}\right)\end{array}$ & $\left|\operatorname{Align}\left(B_{4}^{J}\right)\right|$ & $\begin{array}{l}= \\
= \\
=\end{array}$ & $\left|\begin{array}{l}\mathrm{NC}\left(B_{4}^{J}\right) \\
\operatorname{SW}\left(B_{4}^{J}\right) \\
\mathrm{NN}\left(B_{4}^{J}\right)\end{array}\right|$ \\
\hline\{\} & & 42 & & & 70 & \\
\hline$\left\{s_{1}\right\}$ & & 28 & & & 50 & \\
\hline$\left\{s_{2}\right\}$ & & 32 & & & 58 & \\
\hline$\left\{s_{3}\right\}$ & & 32 & & & 60 & \\
\hline$\left\{s_{4}\right\}$ & & 28 & & & 56 & \\
\hline$\left\{s_{1}, s_{2}\right\}$ & & 14 & & & 30 & \\
\hline$\left\{s_{1}, s_{3}\right\}$ & & 22 & & & 44 & \\
\hline$\left\{s_{1}, s_{4}\right\}$ & & 19 & & & 41 & \\
\hline$\left\{s_{2}, s_{3}\right\}$ & & 17 & & & 40 & \\
\hline$\left\{s_{2}, s_{4}\right\}$ & & 22 & & & 48 & \\
\hline$\left\{s_{3}, s_{4}\right\}$ & & 14 & & & 28 & \\
\hline$\left\{s_{1}, s_{2}, s_{3}\right\}$ & & 5 & & & 16 & \\
\hline$\left\{s_{1}, s_{2}, s_{4}\right\}$ & & 10 & & & 26 & \\
\hline$\left\{s_{1}, s_{3}, s_{4}\right\}$ & & 10 & & & 22 & \\
\hline$\left\{s_{2}, s_{3}, s_{4}\right\}$ & & 5 & & & 8 & \\
\hline$\left\{s_{1}, s_{2}, s_{3}, s_{4}\right\}$ & & 1 & & & 1 & \\
\hline
\end{tabular}

Table 1: The numbers $\operatorname{Cat}\left(W^{J}\right)$ for $W \in\left\{A_{4}, B_{4}\right\}$. For $B_{4}$ the noncommuting simple reflections satisfy $\left(s_{1} s_{2}\right)^{3}=\left(s_{2} s_{3}\right)^{3}=\left(s_{3} s_{4}\right)^{4}=e$. We have suppressed the dependence of $\left|\operatorname{Align}\left(W^{J}, c\right)\right|,\left|\mathrm{NC}\left(W^{J}, c\right)\right|$ and $\left|\mathrm{SW}\left(W^{J}, c\right)\right|$ on $c$, as they agree for all Coxeter elements.

In studying these tables, we observe that for the groups $A_{4}, B_{4}, H_{3}$ (and trivially for the dihedral groups) there seem to exist well-defined parabolic Coxeter-Catalan numbers. Further computer experiments suggest the following conjecture.

Conjecture 41. Let $(W, S)$ be a Coxeter system with $W \in\left\{A_{n}, B_{n}, H_{3}, I_{2}(m)\right\}$, and let $J \subseteq S$. For any Coxeter element $c \in W$ the cardinalities of the sets $\operatorname{Align}\left(W^{J}, c\right)$, $\mathrm{NC}\left(W^{J}, c\right), \mathrm{SW}\left(W^{J}, c\right)$, and $\mathrm{NN}\left(W^{J}\right)$ are equal, and hence do not depend on the choice of $c$. 


\begin{tabular}{|c|c|c|c|c|c|}
\hline \multirow[t]{2}{*}{$J$} & \multicolumn{2}{|c|}{$\left|\operatorname{Align}\left(D_{4}^{J}, c\right)\right|$} & $\left(D_{4}^{J}, c\right)$ & $=\left|\mathrm{SW}\left(D_{4}^{J}, c\right)\right|$ & \multirow[t]{2}{*}{$\mathrm{NN}\left(D_{4}^{J}\right)$} \\
\hline & $s_{2} s_{3} s_{4} s_{1}$ & $s_{1} s_{2} s_{3} s_{4}$ & $s_{3} s_{2} s_{1} s_{4}$ & $s_{4} s_{2} s_{3} s_{1}$ & \\
\hline\{\} & \multicolumn{4}{|c|}{50} & 50 \\
\hline$\left\{s_{1}\right\}$ & \multicolumn{4}{|c|}{36} & 36 \\
\hline$\left\{s_{2}\right\}$ & \multicolumn{4}{|c|}{42} & 42 \\
\hline$\left\{s_{3}\right\}$ & \multicolumn{4}{|c|}{36} & 36 \\
\hline$\left\{s_{4}\right\}$ & \multicolumn{4}{|c|}{36} & 36 \\
\hline$\left\{s_{1}, s_{2}\right\}$ & 22 & 22 & 21 & 21 & 22 \\
\hline$\left\{s_{1}, s_{3}\right\}$ & \multicolumn{4}{|c|}{27} & 27 \\
\hline$\left\{s_{1}, s_{4}\right\}$ & \multicolumn{4}{|c|}{27} & 27 \\
\hline$\left\{s_{2}, s_{3}\right\}$ & 22 & 21 & 22 & 21 & 22 \\
\hline$\left\{s_{2}, s_{4}\right\}$ & 22 & 21 & 21 & 22 & 22 \\
\hline$\left\{s_{3}, s_{4}\right\}$ & \multicolumn{4}{|c|}{27} & 27 \\
\hline$\left\{s_{1}, s_{2}, s_{3}\right\}$ & \multicolumn{4}{|c|}{8} & 8 \\
\hline$\left\{s_{1}, s_{2}, s_{4}\right\}$ & \multicolumn{4}{|c|}{8} & 8 \\
\hline$\left\{s_{1}, s_{3}, s_{4}\right\}$ & \multicolumn{4}{|c|}{21} & 21 \\
\hline$\left\{s_{2}, s_{3}, s_{4}\right\}$ & \multicolumn{4}{|c|}{8} & 8 \\
\hline$\left\{s_{1}, s_{2}, s_{3}, s_{4}\right\}$ & \multicolumn{4}{|c|}{1} & 1 \\
\hline
\end{tabular}

Table 2: The various numbers $\operatorname{Cat}\left(D_{4}^{J}\right)$. Here $s_{2}$ is the unique simple reflection that does not commute with the other simple reflections. The values of $\left|\operatorname{Align}\left(D_{4}^{J}, c\right)\right|=$ $\left|\mathrm{NC}\left(D_{4}^{J}, c\right)\right|=\left|\mathrm{SW}\left(D_{4}^{J}, c\right)\right|$ are equal for $c$ and $c^{-1}$.

The groups appearing in Conjecture 41 are sometimes called "coincidental types", because they share remarkable features that distinguish them from the other finite Coxeter groups. Some of these features are listed in [11, Theorems 8.5 and 10.2], [17, Theorem 14], [18, Theorem 2], [26], and [32, Remark 3.1.26]. Since the families of parabolic aligned elements, parabolic noncrossing and nonnesting partitions are equinumerous for these groups, we are tempted to define a parabolic Coxeter-Catalan number as follows.

Definition 42. Let $(W, S)$ be a Coxeter system with $W \in\left\{A_{n}, B_{n}, H_{3}, I_{2}(m)\right\}$, and let $J \subseteq S$. Define the parabolic Coxeter-Catalan number by

$$
\operatorname{Cat}\left(W^{J}\right)=\left|\mathrm{NN}\left(W^{J}\right)\right|
$$

\subsection{Aligned Elements for Arbitrary Reduced Expressions}

Observe that the definition of the $\left(W^{J}, c\right)$-aligned elements from Definition 33 does not so much depend on the fact that we consider a parabolic quotient of a Coxeter group, rather than on the particular reduced expression of $w_{\circ}^{J}$ we have chosen. More precisely, the alignment property depends on the inversion order $\operatorname{Inv}\left(\mathbf{w}_{\circ}^{\mathbf{J}}\right)$. This suggests the following definition. 


\begin{tabular}{|c|c|c|}
\hline$J$ & $\left|\operatorname{Align}\left(H_{3}^{J}\right)\right|=\left|\mathrm{NC}\left(H_{3}^{J}\right)\right|=\left|\mathrm{SW}\left(H_{3}^{J}\right)\right|=\left|\mathrm{NN}\left(H_{3}^{J}\right)\right|$ \\
\hline\{\} & 32 \\
$\left\{s_{1}\right\}$ & 27 \\
$\left\{s_{2}\right\}$ & 28 \\
$\left\{s_{3}\right\}$ & 25 \\
$\left\{s_{1}, s_{2}\right\}$ & 12 \\
$\left\{s_{1}, s_{3}\right\}$ & 22 \\
$\left\{s_{2}, s_{3}\right\}$ & 18 \\
$\left\{s_{1}, s_{2}, s_{3}\right\}$ & 1 \\
\hline
\end{tabular}

Table 3: The numbers $\operatorname{Cat}\left(H_{3}^{J}\right)$, where the noncommuting simple reflections satisfy $\left(s_{1} s_{2}\right)^{5}=\left(s_{2} s_{3}\right)^{3}=e$. As in Figure 1, we have suppressed the dependence of $\left|\operatorname{Align}\left(H_{3}^{J}, c\right)\right|,\left|\mathrm{NC}\left(H_{3}^{J}, c\right)\right|$ and $\left|\mathrm{SW}\left(H_{3}^{J}, c\right)\right|$ on $c$.

Definition 43. Let $(W, S)$ be a Coxeter system, let $w \in W$, and fix a reduced expression $\mathbf{w}$ for $w$. An element $x \leqslant_{S} w$ is $\mathbf{w}$-aligned if whenever we have $t_{\alpha}<t_{a \alpha+b \beta}<t_{\beta}$ in the inversion order $\operatorname{Inv}(\mathbf{w})$ for $a, b$ positive integers, then $t_{a \alpha+b \beta} \in \operatorname{Cov}(x)$ implies $t_{\alpha} \in \operatorname{Inv}(x)$.

In particular, this definition requires that $t_{\alpha}, t_{a \alpha+b \beta}, t_{\beta} \in \operatorname{Inv}(w)$. Let $\operatorname{Align}(W, \mathbf{w})$ denote the set of all $\mathbf{w}$-aligned elements of $W$. Note that at this level of generality we do not even need to require that $W$ is finite, and we can pick any element $w \in W$. It is immediate that if $W$ is finite and $c \in W$ is a Coxeter element, then $x \in W$ is $c$-aligned if and only if it is $\mathbf{w}_{\circ}(\mathbf{c})$-aligned. Let us illustrate Definition 43 with an example.

Example 44. Let $W=\tilde{A}_{3}$ be the affine symmetric group of rank 4 . Denote its simple reflections by $s_{0}, s_{1}, s_{2}, s_{3}$ such that the following Coxeter relations hold:

$$
\left(s_{0} s_{1}\right)^{3}=\left(s_{1} s_{2}\right)^{3}=\left(s_{2} s_{3}\right)^{3}=\left(s_{0} s_{3}\right)^{3}=\left(s_{0} s_{2}\right)^{2}=\left(s_{1} s_{3}\right)^{2}=e .
$$

Pick $w \in \tilde{A}_{3}$ given by the reduced expression $\mathbf{w}=s_{0} s_{1} s_{0} s_{3} s_{0} s_{1} s_{2}$. The weak order interval $[e, w]$ is shown in Figure 5. The inversion $\operatorname{order} \operatorname{Inv}(\mathbf{w})$ is given by

$$
s_{0}<s_{0} s_{1} s_{0}<s_{1}<s_{1} s_{0} s_{3} s_{0} s_{1}<s_{0} s_{3} s_{0}<s_{3}<s_{1} s_{0} s_{3} s_{0} s_{1} s_{2} s_{1} s_{0} s_{3} s_{0} s_{1} .
$$

Let us denote these reflections by $t_{1}, t_{2}, t_{3}, t_{4}, t_{5}, t_{6}, t_{7}$ in that order; and let $\beta_{i}$ be the positive root corresponding to $t_{i}$ for $i \in[7]$. The roots $\beta_{1}, \beta_{3}, \beta_{6}$ are simple; and we have the following decompositions:

$$
\beta_{2}=\beta_{1}+\beta_{3}, \quad \beta_{4}=\beta_{2}+\beta_{6}=\beta_{3}+\beta_{5}, \quad \beta_{5}=\beta_{1}+\beta_{6} .
$$

The root $\beta_{7}$ is not simple, but cannot be written as a (nontrivial) linear combination of any of the $\beta_{i}$ 's. In view of Definition 43 an element $x \leqslant_{S} w$ is $\mathbf{w}$-aligned if whenever it has $t_{2}$, or $t_{4}$, or $t_{5}$ as a cover reflection, then it needs to have $t_{1}$, or $t_{2}$ and $t_{3}$, or $t_{1}$, respectively, as inversions. This is satisfied for the elements in Figure 5 highlighted in gray. If we consider $x=s_{1} s_{0} s_{3} s_{0}$, then we can check that $\operatorname{Cov}(x)=\left\{t_{2}, t_{6}\right\}$ and $\operatorname{Inv}(x)=\left\{t_{2}, t_{3}, t_{4}, t_{6}\right\}$. Therefore, $x$ is not $\mathbf{w}$-aligned. 


\begin{tabular}{|c|c|c|c|}
\hline$J$ & $\left|\operatorname{Align}\left(H_{4}^{J}\right)\right|=\left|\mathrm{NC}\left(H_{4}^{J}\right)\right|=\left|\mathrm{SW}\left(H_{4}^{J}\right)\right|$ & $\left|\mathrm{NN}\left(H_{4}^{J}\right)\right|$ \\
\hline\{\} & 280 & 280 \\
$\left\{s_{1}\right\}$ & 266 & 266 \\
$\left\{s_{2}\right\}$ & 270 & 270 \\
$\left\{s_{3}\right\}$ & 266 & 266 \\
$\left\{s_{4}\right\}$ & 248 & 248 \\
$\left\{s_{1}, s_{2}\right\}$ & 209 & 210 \\
$\left\{s_{1}, s_{3}\right\}$ & 256 & 256 \\
$\left\{s_{1}, s_{4}\right\}$ & 239 & 239 \\
$\left\{s_{2}, s_{3}\right\}$ & 245 & 245 \\
$\left\{s_{2}, s_{4}\right\}$ & 242 & 242 \\
$\left\{s_{3}, s_{4}\right\}$ & 216 & 216 \\
$\left\{s_{1}, s_{2}, s_{3}\right\}$ & 95 & 106 \\
$\left\{s_{1}, s_{2}, s_{4}\right\}$ & 197 & 198 \\
$\left\{s_{1}, s_{3}, s_{4}\right\}$ & 212 & 212 \\
$\left\{s_{2}, s_{3}, s_{4}\right\}$ & 191 & 191 \\
$\left\{s_{1}, s_{2}, s_{3}, s_{4}\right\}$ & 1 & 1 \\
\hline
\end{tabular}

Table 4: The various numbers $\operatorname{Cat}\left(H_{4}^{J}\right)$, where the noncommuting simple reflections satisfy $\left(s_{1} s_{2}\right)^{5}=\left(s_{2} s_{3}\right)^{3}=\left(s_{3} s_{4}\right)^{3}=e$. As in Figure 1, we have suppressed the dependence of $\left|\operatorname{Align}\left(H_{4}^{J}, c\right)\right|,\left|\mathrm{NC}\left(H_{4}^{J}, c\right)\right|$ and $\left|\mathrm{SW}\left(H_{4}^{J}, c\right)\right|$ on $c$. The values for $\left|\mathrm{NN}\left(H_{4}^{J}\right)\right|$ were computed using the four candidate "root posets" in Figure 5 of [9], all of which gave the same numbers.

It is tempting to conjecture that the weak order on $\mathbf{w}$-aligned elements always forms a lattice (and therefore to extend Conjecture 35 to the more general setting of Definition 43). However, this turns out to be false, even in finite type. If we take $W=A_{4}$ and $w \in W$ given by the reduced expression $\mathbf{w}=s_{2} s_{1} s_{2} s_{3} s_{4} s_{2} s_{1}$, then there are twenty $\mathbf{w}$-aligned elements, but Weak $\left(\operatorname{Align}\left(A_{4}, \mathbf{w}\right)\right)$ is not a lattice. So far, Example 44 shows the smallest poset of $\mathbf{w}$-aligned elements known to us that is not a lattice under weak order. (Note that the elements $s_{0} s_{1} s_{0} s_{3} s_{0} s_{1}$ and $s_{1} s_{0} s_{3} s_{0} s_{1} s_{2}$ have two maximal $\mathbf{w}$-aligned lower bounds, namely $s_{1} s_{0} s_{3}$ and $s_{1} s_{3}$.)

We have not been able to determine necessary and sufficient conditions on $W$ and w such that $\operatorname{Weak}(\operatorname{Align}(W, \mathbf{w}))$ is a lattice. It turns out that the next best candidate, namely the conjecture that $\operatorname{Weak}(\operatorname{Align}(W, \mathbf{w}(\mathbf{c})))$ for some Coxeter element $c$ is always a lattice, is also wrong. Consider again $W=A_{4}$ and $\mathbf{w}=s_{3} s_{4} s_{1} s_{3} s_{2} s_{1} s_{3} s_{4}$. This is a $s_{3} s_{4} s_{2} s_{1}$-sorting word, but the corresponding weak order poset is not a lattice.

We are, however, not aware of any counterexamples in rank 3.

\section{Acknowledgements}

The second author would like to thank D. Stanton, V. Reiner, and H. Thomas for their support and guidance, N. Reading and C. Ceballos for helpful conversations, and C. Ar- 


\begin{tabular}{|c|c|c|c|c|c|}
\hline \multirow[t]{2}{*}{$J$} & \multicolumn{2}{|c|}{$\left|\operatorname{Align}\left(F_{4}^{J}, c\right)\right|$} & $\mathrm{C}\left(F_{4}^{J}, c\right)$ & $\mathrm{SW}\left(F_{4}^{J}, c\right)$ & \multirow[t]{2}{*}{$\mathrm{NN}\left(F_{4}^{J}\right) \mid$} \\
\hline & $s_{2} s_{3} s_{4} s_{1}$ & $s_{1} s_{2} s_{3} s_{4}$ & $s_{3} s_{2} s_{1} s_{4}$ & $s_{4} s_{2} s_{3} s_{1}$ & \\
\hline\{\} & \multicolumn{4}{|c|}{105} & 105 \\
\hline$\left\{s_{1}\right\}$ & \multicolumn{4}{|c|}{85} & 85 \\
\hline$\left\{s_{2}\right\}$ & \multicolumn{4}{|c|}{95} & 95 \\
\hline$\left\{s_{3}\right\}$ & \multicolumn{4}{|c|}{95} & 95 \\
\hline$\left\{s_{4}\right\}$ & \multicolumn{4}{|c|}{85} & 85 \\
\hline$\left\{s_{1}, s_{2}\right\}$ & \multicolumn{4}{|c|}{65} & 65 \\
\hline$\left\{s_{1}, s_{3}\right\}$ & \multicolumn{4}{|c|}{79} & 79 \\
\hline$\left\{s_{1}, s_{4}\right\}$ & \multicolumn{4}{|c|}{71} & 71 \\
\hline$\left\{s_{2}, s_{3}\right\}$ & 62 & 57 & 62 & 62 & 63 \\
\hline$\left\{s_{2}, s_{4}\right\}$ & \multicolumn{4}{|c|}{79} & 79 \\
\hline$\left\{s_{3}, s_{4}\right\}$ & \multicolumn{4}{|c|}{65} & 65 \\
\hline$\left\{s_{1}, s_{2}, s_{3}\right\}$ & 23 & 23 & 23 & 23 & 24 \\
\hline$\left\{s_{1}, s_{2}, s_{4}\right\}$ & \multicolumn{4}{|c|}{57} & 57 \\
\hline$\left\{s_{1}, s_{3}, s_{4}\right\}$ & \multicolumn{4}{|c|}{57} & 57 \\
\hline$\left\{s_{2}, s_{3}, s_{4}\right\}$ & \multicolumn{4}{|c|}{23} & 23 \\
\hline$\left\{s_{1}, s_{2}, s_{3}, s_{4}\right\}$ & \multicolumn{4}{|c|}{1} & 1 \\
\hline
\end{tabular}

Table 5: The various numbers $\operatorname{Cat}\left(F_{4}^{J}\right)$. Here we have the relations $\left(s_{1} s_{2}\right)^{3}=\left(s_{2} s_{3}\right)^{4}=$ $\left(s_{3} s_{4}\right)^{3}=e$ between noncommuting simple reflections. The values of $\left|\operatorname{Align}\left(W^{J}, c\right)\right|=$ $\left|\mathrm{NC}\left(W^{J}, c\right)\right|=\left|\mathrm{SW}\left(F_{4}^{J}, c\right)\right|$ are equal for $c$ and $c^{-1}$.

reche for his proofreading.

\section{References}

[1] D. Armstrong, Generalized noncrossing partitions and combinatorics of Coxeter groups, Mem. Amer. Math. Soc. 202 (2009).

[2] D. Armstrong, C. Stump, and H. Thomas, A uniform bijection between nonnesting and noncrossing partitions, Trans. Amer. Math. Soc. 365 (2013), 4121-4151.

[3] C. A. Athanasiadis, On noncrossing and nonnesting partitions for classical reflection groups, Electron. J. Combin. (1998).

[4] D. Bessis, The dual braid monoid, Ann. Sci. École Norm. Sup. 36 (2003), 647-683.

[5] A. Björner and F. Brenti, Combinatorics of Coxeter Groups, Springer, New York, 2005.

[6] A. Björner and M. L. Wachs, Generalized quotients in Coxeter groups, Trans. Amer. Math. Soc. 308 (1988), 1-37.

[7] A. Björner and M. L. Wachs, Shellable and nonpure complexes and posets II, Trans. Amer. Math. Soc. 349 (1997), 3945-3975. 


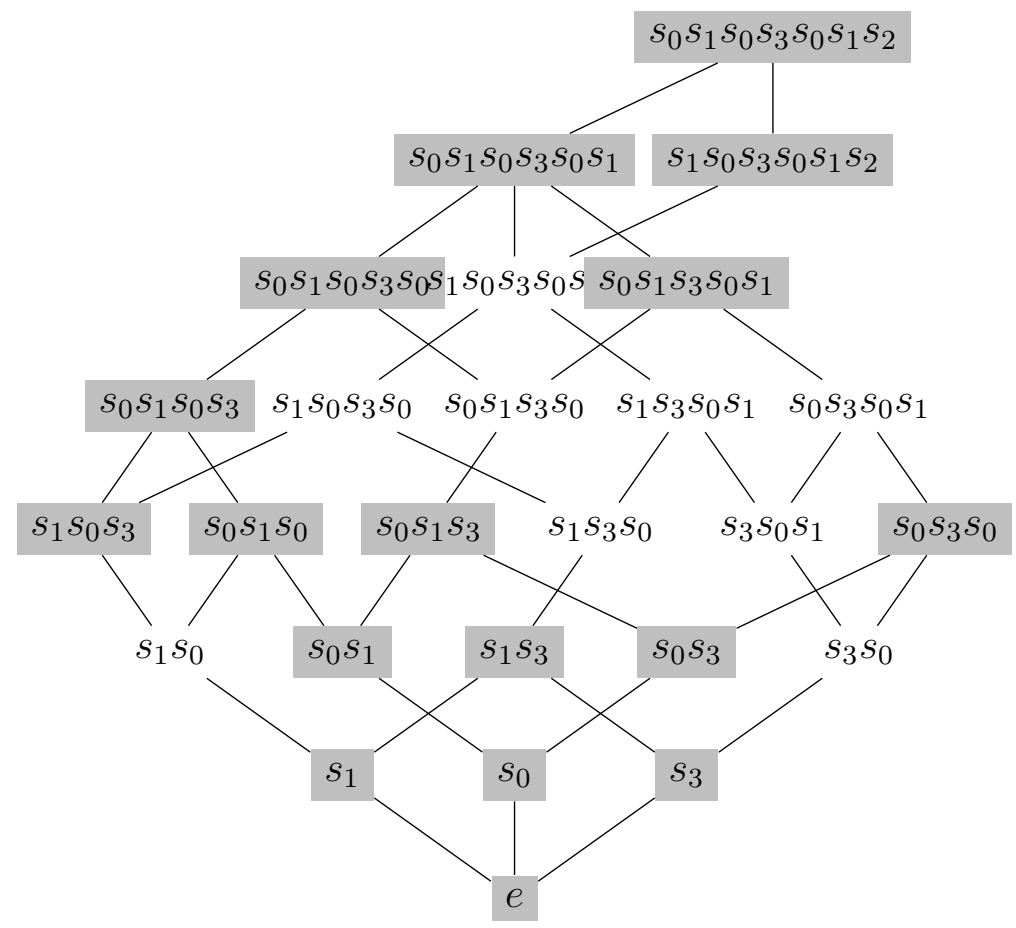

Figure 5: The weak order interval $[e, w]$, where $w$ is given by the reduced expression $\mathbf{w}=$ $s_{0} s_{1} s_{0} s_{3} s_{0} s_{1} s_{2}$ in the affine Coxeter group $\tilde{A}_{3}$. The $\mathbf{w}$-aligned elements are highlighted in gray.

[8] T. Brady and C. Watt, Non-crossing partition lattices in finite real reflection groups, Trans. Amer. Math. Soc. 360 (2008), 1983-2005.

[9] M. Cuntz and C. Stump, On root posets for noncrystallographic root systems, Math. Comp. 84 (2015), 485-503.

[10] M. Dyer, On the weak order of Coxeter groups, Can. J. Math. 71 (2019), 299-336.

[11] S. Fomin and N. Reading, Generalized cluster complexes and Coxeter combinatorics, Int. Math. Res. Notes 44 (2005), 2709-2757.

[12] G. Grätzer, Lattice Theory: Foundation, Springer, Basel, 2011.

[13] J. E. Humphreys, Reflection Groups and Coxeter Groups, Cambridge University Press, Cambridge, 1990.

[14] A. Knutson and E. Miller, Subword complexes in Coxeter groups, Adv. Math. 184 (2004), 161-176.

[15] G. Kreweras, Sur une classe de problèmes de dénombrement liés au treillis des partitions des entiers, Cahiers du B.U.R.O. Univ. de Rech. Opér. 6 (1965), 5-105.

[16] G. Kreweras, Sur les partitions non croisées d'un cycle, Discrete Math. 1 (1972), 333-350. 
[17] A. R. Miller, Foulkes characters for complex reflection groups, Proc. Amer. Math. Soc. 143 (2015), 3281-3293.

[18] H. Mühle, SB-labelings, distributivity, and Bruhat order on sortable elements, Electron. J. Combin. 22 (2015).

[19] H. Mühle and N. Williams, Tamari lattices for parabolic quotients of the symmetric group, Discrete Math. Theor. Comput. Sci. (2015), 973-984.

[20] R. A. Proctor and M. J. Willis, Parabolic Catalan numbers count efficient inputs for Gessel-Viennot flagged Schur function determinant (2017). arXiv:1701.01182.

[21] R. A. Proctor and M. J. Willis, Parabolic Catalan numbers count flagged Schur functions and their appearances as type A Demazure characters (key polynomials), Discrete Math. Theor. Comput. Sci. 19 (2017).

[22] R. A. Proctor and M. J. Willis, Convexity of tableau sets for type A Demazure characters (key polynomials), parabolic Catalan numbers, Discrete Math. Theor. Comput. Sci. 20 (2018).

[23] N. Reading, Cambrian lattices, Adv. Math. 205 (2006), 313-353.

[24] N. Reading, Clusters, Coxeter-sortable elements and noncrossing partitions, Trans. Amer. Math. Soc. 359 (2007), 5931-5958.

[25] N. Reading, Sortable elements and cambrian lattices, Algebra Universalis 56 (2007), 411-437.

[26] N. Reading, Chains in the noncrossing partition lattice, SIAM J. Discrete Math. 22 (2008), 875-886.

[27] N. Reading, Noncrossing arc diagrams and canonical join representations, SIAM J. Discrete Math. 29 (2015), 736-750.

[28] V. Reiner, Non-crossing partitions for classical reflection groups, Discrete Math. 177 (1997), 195-222.

[29] L. Serrano and C. Stump, Maximal fillings of moon polyominoes, simplicial complexes, and Schubert polynomials, Electron. J. Combin. 19 (2012).

[30] J. R. Stembridge, On the fully commutative elements of Coxeter groups, J. Algebraic Combin. 5 (1996), 353-385.

[31] D. Tamari, Monoides préordonnés et chaînes de Malcev, Thèse de Mathématiques, Université de Paris, 1951.

[32] N. Williams, Cataland, Dissertation, University of Minnesota, 2013. 